Open Access

\title{
Tomato S/3-MMP, a member of the Matrix metalloproteinase family, is required for disease resistance against Botrytis cinerea and Pseudomonas syringae pv. tomato DC3000
}

Dayong Li, Huijuan Zhang, Qiuming Song, Lu Wang, Shixia Liu, Yongbo Hong, Lei Huang and Fengming Song*

\begin{abstract}
Background: Matrix metalloproteinases (MMPs) are a family of zinc-dependent endopeptidases. MMPs have been characterized in detail in mammals and shown to play key roles in many physiological and pathological processes. Although MMPs in some plant species have been identified, the function of MMPs in biotic stress responses remains elusive.

Results: A total of five MMP genes were identified in tomato genome. qRT-PCR analysis revealed that expression of SI-MMP genes was induced with distinct patterns by infection of Botrytis cinerea and Pseudomonas syringae pv. tomato (Pst) DC3000 and by treatment with defense-related hormones such as salicylic acid, jasmonic acid and ethylene precursor 1-amino cyclopropane-1-carboxylic acid. Virus-induced gene silencing (VIGS)-based knockdown of individual SI-MMPS and disease assays indicated that silencing of S/3-MMP resulted in reduced resistance to $B$. cinerea and Pst DC3000, whereas silencing of other four SI-MMPs did not affect the disease resistance against these two pathogens. The SI3-MMP-silenced tomato plants responded with increased accumulation of reactive oxygen species and alerted expression of defense genes after infection of B. cinerea. Transient expression of S/3-MMP in leaves of Nicotiana benthamiana led to an enhanced resistance to $B$. cinerea and upregulated expression of defense-related genes. Biochemical assays revealed that the recombinant mature SI3-MMP protein had proteolytic activities in vitro with distinct preferences for specificity of cleavage sites. The SI3-MMP protein was targeted onto the plasma membrane of plant cells when transiently expressed in onion epidermal cells.
\end{abstract}

Conclusion: VIGS-based knockdown of S/3-MMP expression in tomato and gain-of-function transient expression of S/3-MMP in N. benthamiana demonstrate that S/3-MMP functions as a positive regulator of defense response against $B$. cinerea and Pst DC3000.

Keywords: Tomato (Solanum lycopersicum), Matrix metalloproteinases, Botrytis cinerea, Pseudomonas syringae pv. tomato DC3000, Disease resistance, Proteolysis

\footnotetext{
* Correspondence: fmsong@zju.edu.cn

National Key Laboratory for Rice Biology, Institute of Biotechnology, Zhejiang University, Hangzhou, Zhejiang 310058, China
} 


\section{Background}

Proteases play key roles in the regulation of a variety of biological processes [1]. Matrix metalloproteinases (MMPs) are a family of zinc- and calcium-dependent proteases belonging to the metzincin clan of metalloendopeptidases, EC subclass 3.4.24, MA (M) clan according to the MEROPS database [2,3]. The MMP family is characterized by the presence of a highly conserved catalytic domain containing an HEXXHXXGXX(H/D) zincbinding sequence followed by a conserved methionine that forms a tight 1,4- $\beta$ turn called Met-turn [4]. Members of this family have mainly been studied in mammals, but have also been found in simpler animals and plants [5]. In human, 23 MMP genes have been identified to encode proteins with similar structure, e.g., an $\mathrm{N}$-terminal signal peptide for the secretory pathway, a prodomain that regulates the latency of the enzyme and a catalytic domain with the active zinc-binding site [6]. In addition, most of the human MMP proteins contain a C-terminal hemopexin (HPX)-like domain, which is believed to be important in regulating the activity and specificity of the catalytic domain $[7,8]$. It has been shown that human MMPs play key roles in many physiological and pathological processes $[9,10]$.

Members of the MMP family have been identified in higher plants, but only few of them have been studied to date [2]. Similar to the human MMPs, the predicted primary structures of plant MMPs contain a signal peptide, a prodomain with the cysteine-switch motif and a catalytic domain containing the active zinc-binding sequence and structural zinc- and calcium-binding site followed by the conserved Met-turn [11-13]. Activation of MMPs requires physical delocalization of the prodomain from the catalytic site by proteolytic or nonproteolytic mechanisms [14]. It is believed that all plant MMPs are synthesized as inactive forms and are localized either in the plasma membrane or in the extracellular space. However, it was found that Arabidopsis At4-MMP contains a predicted non-cleavable $\mathrm{N}$-terminal signal peptide and tobacco Nt1-MMP was inserted into the plasma membrane [15].

The biological function of MMP proteases in higher plants is largely unknown. Based on the expression patterns, it is proposed that the plant MMPs may be involved in remodeling of the extracellular matrix (ECM) during plant growth and development [2]. The first plant MMP was identified as an ethylenediaminetetraacetic acid (EDTA)-sensitive Azocoll-degrading enzyme in soybean [16]. In cucumber, Cs1-MMP was found to be associated with senescence and cell death in cotyledon development [17]. In Arabidopsis, $5 M M P$ genes were identified and were found to be differentially expressed in roots, leaves, stems and flowers [15]. The At2-MMP mutant plants exhibited altered growth in association with late flowering and early senescence, supporting a physiological and developmental role for plant MMPs [18]. In Medicago truncatula, expression of Mt1-MMP was induced in young nodules, specifically in association with Sinorhizobium meliloti infection [13]. An Mt1$M M P$ RNAi mutant in $M$. truncatula showed nodules with enlarged infection threads and substantial increase in the number of bacterial colonies; whereas an ectopic overexpression of $M t 1-M M P$ in roots led to a significant decrease in nodule number [13]. On the other hand, several lines of evidence also indicate that MMPs may be involved in biotic and abiotic stress responses in plants. In soybean, Gm2-MMP was isolated as a pathogeninduced gene [19]. Expression of Gm2-MMP was induced rapidly in compatible and incompatible interactions with pathogens, but not by salicylic acid (SA) and jasmonic acid (JA), two classical pathogen response signaling molecules [19]. In the tobacco suspension line BY-2, Nt1-MMP was expressed at low level but was induced immediately after treatment with Pseudomonas syringae [11]. In Arabidopsis, distinct expression patterns for each $M M P$ in response to various abiotic and biotic stresses were described in the Genevestigator analysis [20]. At3-MMP showed significant changes in transcript levels under stress conditions, while other $M M P s$ displayed minimal transcript changes [20]. The expression of At2-MMP is tightly controlled in a tissue-responsive way during stress conditions. At2-MMP in 4-week-old plants was induced in leaves by cadmium or methyl jasmonate and in roots by sodium chloride; however, cadmium inhibited the expression of At2-MMP in inflorescence and leaves of 10-week-old plants [18].

In the present study, we characterized the MMP family in tomato and performed functional analyses for their roles in disease resistance. A total of five MMP genes were identified in tomato and their expression was induced with distinct patterns in response to pathogen infection and treatments with defense-related hormones. Silencing of Sl3-MMP in tomato resulted in reduced resistance to Botrytis cinerea and Pseudomonas syringae pv. tomato (Pst) DC3000 whereas transient expression of Sl3-MMP in Nicotiana benthamiana led to an enhanced resistance to $B$. cinerea. Our data demonstrate that member of the MMP family may participate in the regulation of defense response in plants against pathogen infection.

\section{Results}

\section{Identification of the SI-MMP family in tomato}

To identify members of the MMP family in tomato, HMM and Blastp searches using MMP proteins previously reported from Arabidopsis and other plant species as queries against the recently published tomato genome sequences (Release Version ITAG2.40) were performed. 
Five significant hits corresponding to non-redundant putative Sl-MMP genes were identified (Table 1). All five $S l-M M P s$ are intronless genes, which is consistent with structural features of genes for MMPs in Arabidopsis, soybean, cucumber and Medicago truncatula [13, 15, 17, 19, 21]. Full-length cDNAs for Sl2-MMP and Sl3-MMP were identified in the NCBI and SOL databases while no fulllength cDNA was found for other three members (Table 1). We amplified and cloned all 5 Sl-MMP genes using genespecific primers and confirmed by sequencing. These sequences were submitted to GenBank for deposition and presented in Additional file 1.

The Sl-MMP proteins are approximately 360 amino acids with molecular weight of $\sim 40 \mathrm{kDa}$ (Table 1 ). The Sl-MMP proteins shared conserved structural features, e.g., a signal sequence at $\mathrm{N}$ terminus, a propeptide domain, a catalytic domain, a transmembrane domain at $\mathrm{C}$ terminus (Fig. 1a). Characteristic motifs including a PRCGxxD motif, which is characteristic of the cysteine switch mechanism of activation [22], in the propeptide domain and a HExGHxxGxxH zinc-binding region and a conserved methionine residue in the Met-turn in the catalytic domain are present in the Sl-MMP proteins (Fig. 1b). In addition, each of Sl-MMPs contains an invariant DLESV motif on the $\mathrm{N}$-terminal side of the zinc-binding region (Fig. 1b), which is thought to be a plant-specific motif with unknown function [15]. This motif is replaced by a distinct consensus sequence of NLFLV in human and insect MMPs $[4,23]$ but is not present in single-celled green algae MMPs [20, 24]. In term of secondary structure feature, Sl-MMPs have $2 \beta$ strands, $4 \beta$-sheets and $3 \alpha$-helices and they all contain three active site histidines and a catalytic glutamate residue in the zinc-binding region (Fig. 1a). Putative conserved structural ligands for binding zinc and calcium including 3 histidine (H), 2 aspartic acid (D) and 1 glutamic acid residues are present in Sl-MMPs (Fig. 1a). However, like MMPs from other plants, Sl-MMPs do not contain a C-terminal hemopexin domain, which is present in most human MMPs [8].

Phylogenetic tree analysis of Sl-MMPs with previously identified MMPs from other plant species clearly distinguished different groups, which had distinct features linked to plant species or specific functions. Groups I and II can be further separated into two subgroups, representing MMP branches from dicots and monocots. Sl2-MMP, Sl3-MMP and Sl4-MMP are assigned in Group I, which contain Nt1-MMP, At2-MMP and At3-MMP that are known to be pathogen-responsive $[11,18,20]$. Sl1-MMP and Sl5-MMP belong to Group II, whose members have been proposed to be involved in plant growth and development [20]. Group III only hosts MMPs from legumes such as Glycine max and Medicago truncatula and thus seem to be legume-specific.

\section{Expression of SI-MMPs in response to pathogens and defense signaling-related hormones}

To explore the possible involvement of Sl-MMPs in defense response against pathogen infection, we first analyzed the expression changes of Sl-MMPs after infection with B. cinerea. As shown in Fig. 2a, expression of the $S l-M M P$ genes were induced upon infection of $B$. cinerea but showed distinct expression patterns. Generally, the expression of Sl1-MMP, Sl3-MMP, Sl4-MMP and $S 15-M M P$ was significantly induced with peaks at $48 \mathrm{~h}$ whereas the expression of Sl2-MMP was induced significantly with peaks at $24 \mathrm{~h}$ after infection with $B$. cinerea, as compared with those in the mock-inoculated plants (Fig. 2a). Specifically, the expression levels of Sl1$M M P$, Sl3-MMP and Sl5-MMP in B. cinerea-infected plants showed $>5$ folds of increases over those in the mock-inoculated plants at $48 \mathrm{~h}$ after inoculation (Fig. 2a). The expressions of Sl1-MMP and Sl3-MMP exhibited 45 folds of increases at $24 \mathrm{~h}$ after infection of B. cinerea. It was noted that the expression of Sl1-MMP, Sl4-MMP and SI5-MMP was induced only at $48 \mathrm{~h}$ after infection of $B$. cinerea (Fig. 2a). These results indicate that the Sl$M M P$ genes respond with different dynamics and magnitude of expression after infection of $B$. cinerea.

We next analyzed the expression changes of Sl-MMPs after infection with Pst DC3000. As shown in Fig. 3b, only Sl1-MMP, Sl2-MMP and Sl3-MMP were induced upon infection of Pst DC3000 and again showed different expression patterns. Generally, the expression of Sl1$M M P$ and Sl3-MMP was induced significantly with peaks at $24 \mathrm{~h}$ while the expression of Sl2-MMP was induced significantly with peaks at $12 \mathrm{~h}$ after infection with Pst DC3000, as compared with those in the mockinoculated plants (Fig. 2b). Specifically, the expression of Sl1-MMP and Sl3-MMP in Pst DC3000-infected plants

Table 1 Characterization of tomato SI-MMP genes and proteins

\begin{tabular}{|c|c|c|c|c|c|c|}
\hline Genes & Locus in SOL & Proteins in NCBI & Size (aa) & $\mathrm{MW}(\mathrm{kD})$ & $p l$ & CDNAs in NCBI/SOL \\
\hline S/1-MMP & Solyc08g078550 & XP_010325488 & 356 & 40.17 & 5.20 & - \\
\hline$S / 2-M M P$ & Solyc04g005040 & $\mathrm{CCH} 68443$ & 363 & 40.00 & 5.46 & AK328733/AK327627/ SGN-U573509 \\
\hline$S / 3-M M P$ & Solyc04g005050 & NP_001266203 & 367 & 40.21 & 5.08 & AK322919/ SGN-U573510 \\
\hline SI4-MMP & Solyc05g006360 & XP_010320628 & 357 & 40.50 & 6.00 & - \\
\hline SI5-MMP & Solyc10g018750 & XP_004248606 & 357 & 40.80 & 7.83 & - \\
\hline
\end{tabular}


(A)

\begin{tabular}{|c|c|c|c|c|c|}
\hline $\begin{array}{c}\text { Signal } \\
\text { peptide }\end{array}$ & $\begin{array}{l}\text { Propeptide } \\
\text { domain }\end{array}$ & $\begin{array}{l}\text { Cysteine } \\
\text { Switch }\end{array}$ & $\begin{array}{l}\text { Catalytic } \\
\text { domain }\end{array}$ & $\begin{array}{l}\text { Zinc } \\
\text { binding } \\
\text { domain }\end{array}$ & TM \\
\hline
\end{tabular}

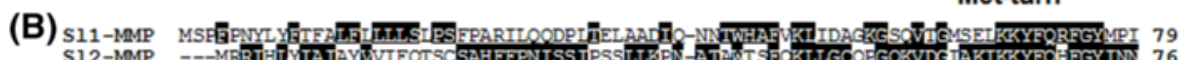

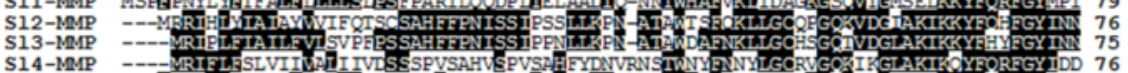

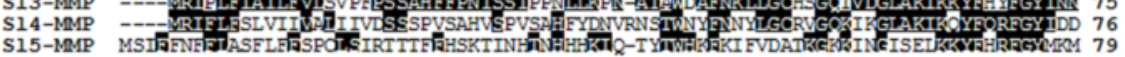

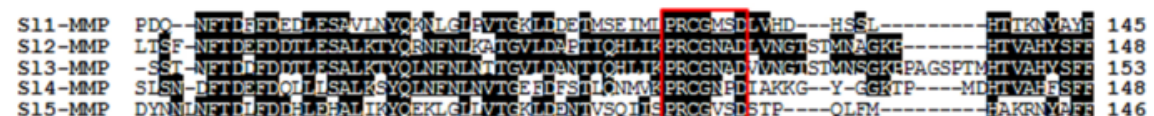

S15-MMP DISN-DFTD FDDPLLSALES
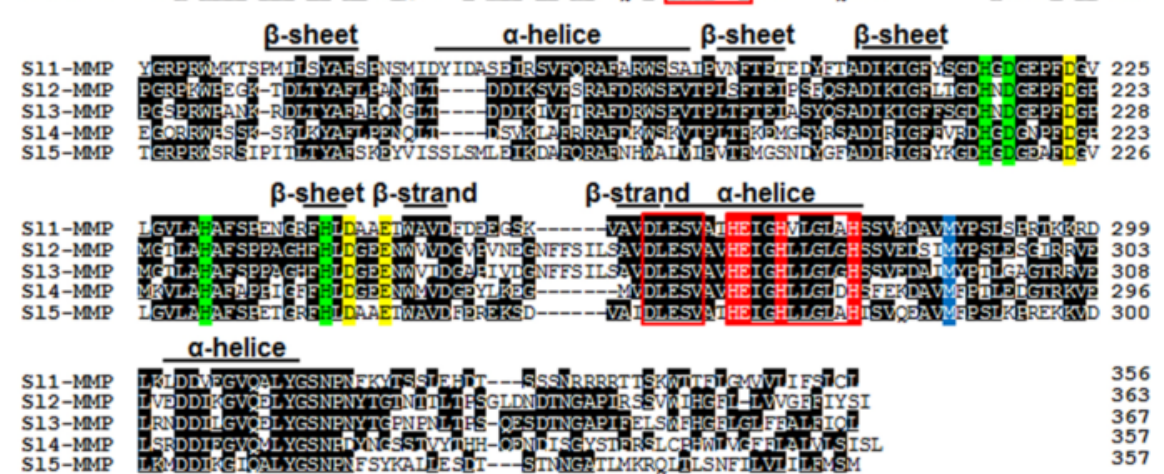

(C)

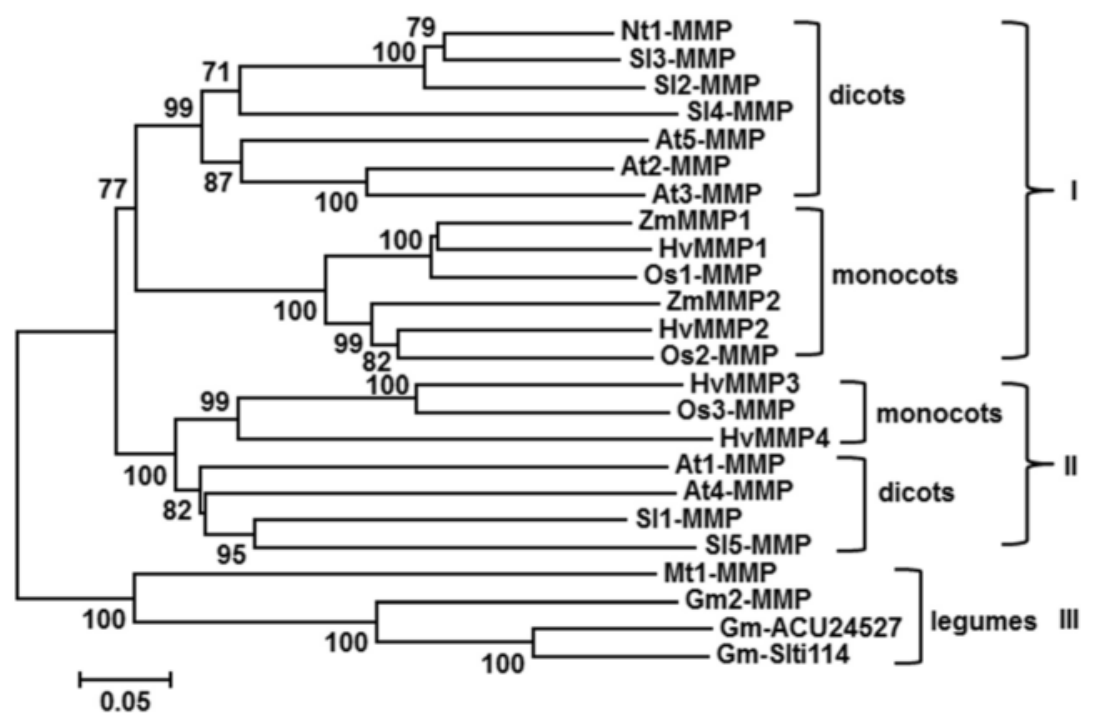

Fig. 1 Sequence alignment and phylogenetic tree analysis of SI-MMP with other plant MMP proteins. a Predicted domains of SI-MMP proteins. TM, transmembrane domain. b Alignment of SI-MMPs. Numbers on the right indicate amino acid positions of the SI-MMP proteins. The cysteine switch motif, zinc-binding sequence and DLESV sequence are boxed in red. Secondary structure features such as $\beta$-strand, $\beta$-sheet and a-helix are indicated above the aligned sequences. The active site histidine and the catalytic glutamate residues are indicated in red. Ligands of the conserved structural zinc and calcium are colored in green and yellow, respectively. The hydrophobic base forming the methionine residue of the Met-turn is highlighted in blue. c Phylogenetic tree analysis of SI-MMPs with other plant MMPs. Phylogenetic tree was constructed by Neighbor-joining method using MEGA program. Plant MMPs used and their GenBank accessions are as follows: Arabidopsis thaliana At1-MMP (NP_193397), At2-MMP (NP__177174), At3-MMP (NP_173824), At4-MMP (NP_182030), At5-MMP (NP_176205), Glycine max Gm2-MMP (AAL27029), Gm-ACU24527 (ACU24527), Gm-Slti1 14 (ABW96008), Hordeum vulgare HvMMP1 (BAJ94792), HvMMP2 (BAJ93963), HvMMP3 (BAJ94176), HvMMP4 (BAJ90264), Medicago truncatula Mt1-MMP (CAA77093), Nicotiana tabacum NtMMP1 (ABF58910), Solanum lycopersicum SI1-MMP (XP_010325488), SI2-MMP (CCH68443), SI3-MMP (NP_001266203), SI4-MMP (XP_010320628), SI5-MMP (XP_004248606), Zea mays ZmMMP1 (NP_001151749), ZmMMP2 (NP_001142095), Oryza sativa Os1-MMP (NP_001048075), Os2-MMP (NP_001057259), Os3-MMP (NP_001065361). Bootstrap values from 100 replicates are indicated at each node. Bar represents the number of amino acid differences per site 


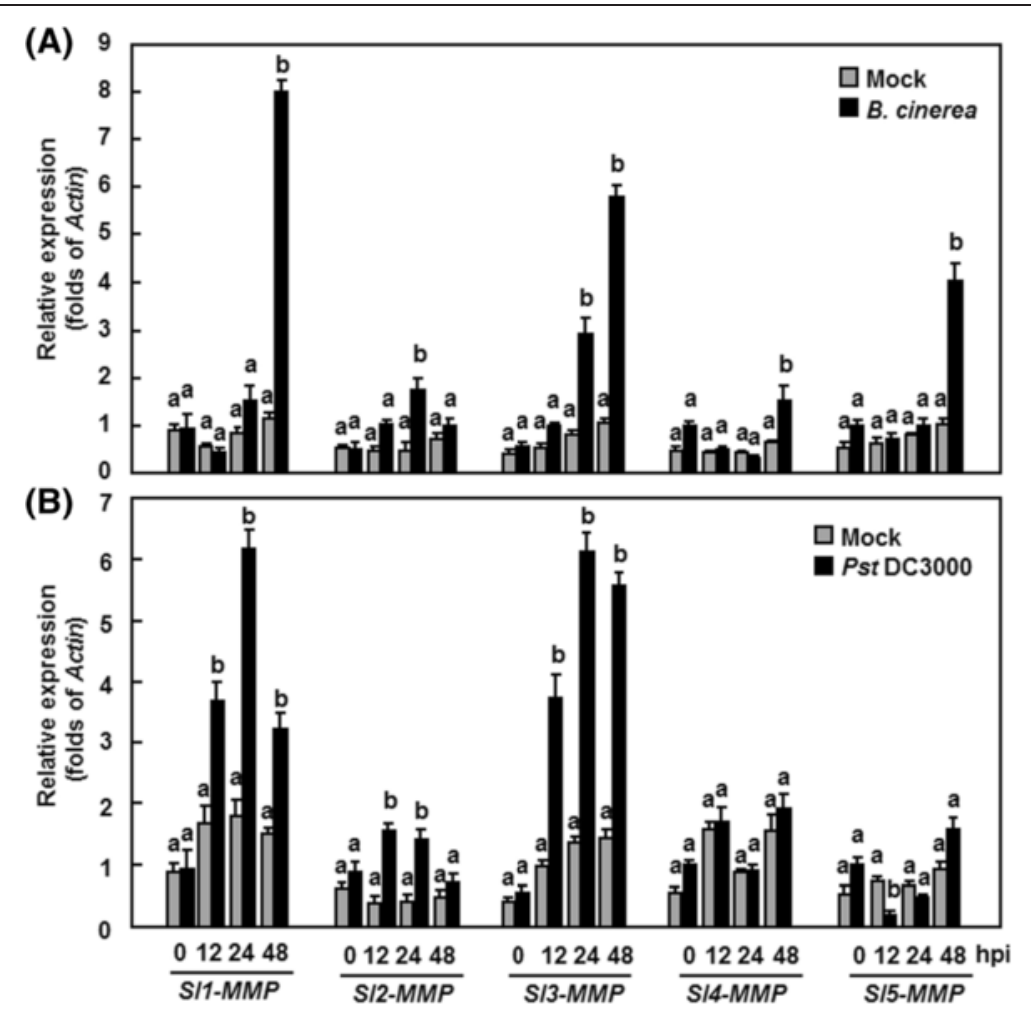

Fig. 2 Expression patterns of SI-MMPs in response to B. cinerea or P. syringae pv. tomato DC3000 treatment. Tomato plants were inoculated by spore suspension $\left(2 \times 10^{5}\right.$ spores $\left./ \mathrm{ml}\right)$ of $B$. cinerea or buffer solution as a mock-inoculation control (a) and by vacuum infiltration with $P$. syringae pv. tomato DC3000 $\left(\mathrm{OD}_{600}=0.0002\right)$ or sterilized $10 \mathrm{mM} \mathrm{MgCl}$ solution as a mock-inoculation control $(\mathbf{b})$. Leaf samples were collected at indicated time points and gene expression was analyzed by qRT-PCR. Relative expression levels were calculated by comparing with the corresponding values at $0 \mathrm{~h}$ (as a control) after inoculation and shown as folds of the actin transcript values. Data presented are the means \pm SD from three independent experiments and different letters above the columns indicate significant differences at $p<0.05$ level

showed $>5$ folds of increases over those in the mockinoculated plants at $24 \mathrm{~h}$, whereas the expression of Sl2$M M P$ was induced significantly with peaks at 12 and $24 \mathrm{~h}$ after infection with Pst DC3000 (Fig. 2b). Interestingly, the expression of $S 15-M M P$ was down-regulated at $12 \mathrm{~h}$ (Fig. 2b). These results indicate that the expression of Sl1-MMP, Sl2-MMP and Sl3-MMP was induced by Pst DC3000.

We also examined the dynamics of Sl-MMPs expressions in tomato plants after treatments with SA, methyl jasmonate (MeJA) and 1-amino cyclopropane-1-carboxylic acid (ACC) [a precursor of ethylene (ET)], three defense signaling-related hormones. As shown in Fig. 4, different expression patterns for Sl-MMPs were observed in response to these defense signaling-related hormones. In SA-treated plants, expression of Sl3-MMP and Sl4-MMP was significantly increased by $2-3$ folds over that in the control plants, while expressions of Sl1-MMP, Sl2-MMP and Sl3-MMP were not affected (Fig. 3a). In JA- or ACCtreated plants, expression of Sl4-MMP was strongly induced, reaching 3-4 folds of increased at $6 \mathrm{~h}$ after treatment (Fig. $3 \mathrm{~b}$ and c). Besides Sl4-MMP, the expression of Sl1-MMP, Sl2-MMP and Sl3-MMP was also induced by JA, showing an increase of 2-3 folds at 12 h (Fig. 3b). Except Sl4-MMP, expression of other four Sl-MMPs was not affected by ACC (Fig. 3c). Interestingly, the expression of Sl5-MMP was not affected by both JA and ACC during the experimental period (Fig. 3b and c). These data indicate that the tomato $S l-M M P s$ respond with different expression patterns to SA, JA and ET, three well-known defense signaling-related hormones.

\section{Silencing of $S / 3-M M P$ resulted in reduced resistance to $B$. cinerea and Pst DC3000}

To examine the possible involvement of Sl-MMPs in disease resistance, we performed functional analyses by virus-induced gene silencing (VIGS) approach through comparing the disease phenotype between individual Sl$M M P$-silenced plants and non-silenced control plants. For this purpose, specific fragment for each Sl-MMP gene (Additional file 2) was chosen to generate VIGS construct and standard VIGS procedure with a phytoene desaturase (PDS) construct as an indicative for VIGS efficiency of each experiment was performed on 2-weekold plants $[25,26]$. Under our experiment conditions, $>90 \%$ 

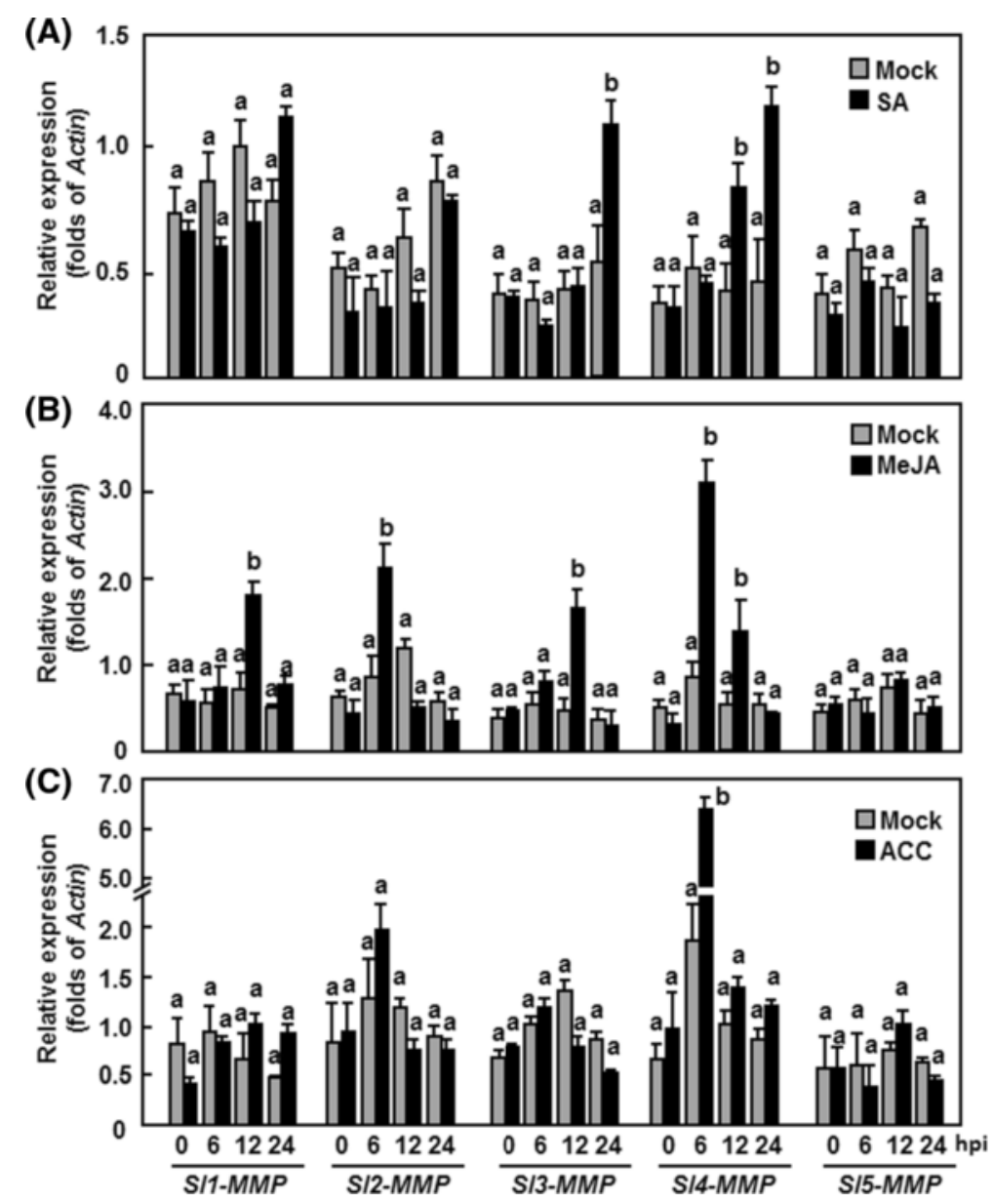

Fig. 3 Expression patterns of SI-MMPs in response to defense signaling hormones. Tomato plants were treated by foliar spraying of $1 \mathrm{mM}$ SA (a), $100 \mu \mathrm{M}$ MeJA (b), $100 \mu \mathrm{M}$ ACC (c) or equal volume of solution as a control and leaf samples were collected at indicated time points. Gene expression was analyzed by qRT-PCR and relative expression levels were calculated by comparing with the corresponding values at $0 \mathrm{~h}$ (as a control) after treatment. Relative expression was shown as folds of the actin transcript values. Data presented are the means \pm SD from three independent experiments and different letters above the columns indicate significant differences at $p<0.05$ level

of the pTRV2-PDS-infiltrated plants showed bleaching phenotype (data not shown). The silencing efficiency and specificity for each $S l-M M P$ gene was examined by qRTPCR analyzing the transcript level of the target Sl-MMP gene and other four Sl-MMP genes in the pTRV2-target Sl$M M P$-infiltrated plants. When compared with those in the pTRV2-GUS-infiltrated plants, the transcript level of the target $S l-M M P$ gene was significantly reduced whereas the transcript levels of the other Sl-MMP genes were comparable in the pTRV2-target Sl-MMP-silenced plants (Fig. 4). Overall, the silencing efficiency for a target $S l-M M P$ gene was approximately $70 \%$ (Fig. 4). The efficiencies and specificity of silencing for each individual Sl-MMP gene were satisfied for further experiments and all the subsequent experiments were performed only on those pTRV2-SlMMPs-infiltrated plants with high levels of silencing efficiency $(>70 \%)$.
We first examined the possible involvement of $\mathrm{Sl}$ $M M P s$ in resistance against $B$. cinerea by challenging the pTRV2-Sl-MMPs-infiltrated plants with spore suspension of B. cinerea and comparing the disease severity and in planta fungal growth with those in pTRV-GUSinfiltrated non-silenced plants. In our detached leaf assays, B. cinerea-caused lesions on detached leaves from the pTRV2-Sl1-MMP-, pTRV2-Sl2-MMP-, pTRV2-Sl4MMP- and pTRV2-S15-MMP-infiltrated plants were similar to those on the detached leaves from pTRV2GUS-infilrtratd plants (Fig. 5a), suggesting that Sl1$M M P$, Sl2-MMP, Sl4-MMP and Sl51-MMP may not be involved in resistance against $B$. cinerea. However, $B$. cinerea-caused lesions on detached leaves from the pTRV2-Sl3-MMP-infiltrated plans were significantly larger and developed faster, merging into large necrotic areas, as compared with those on leaves from the 


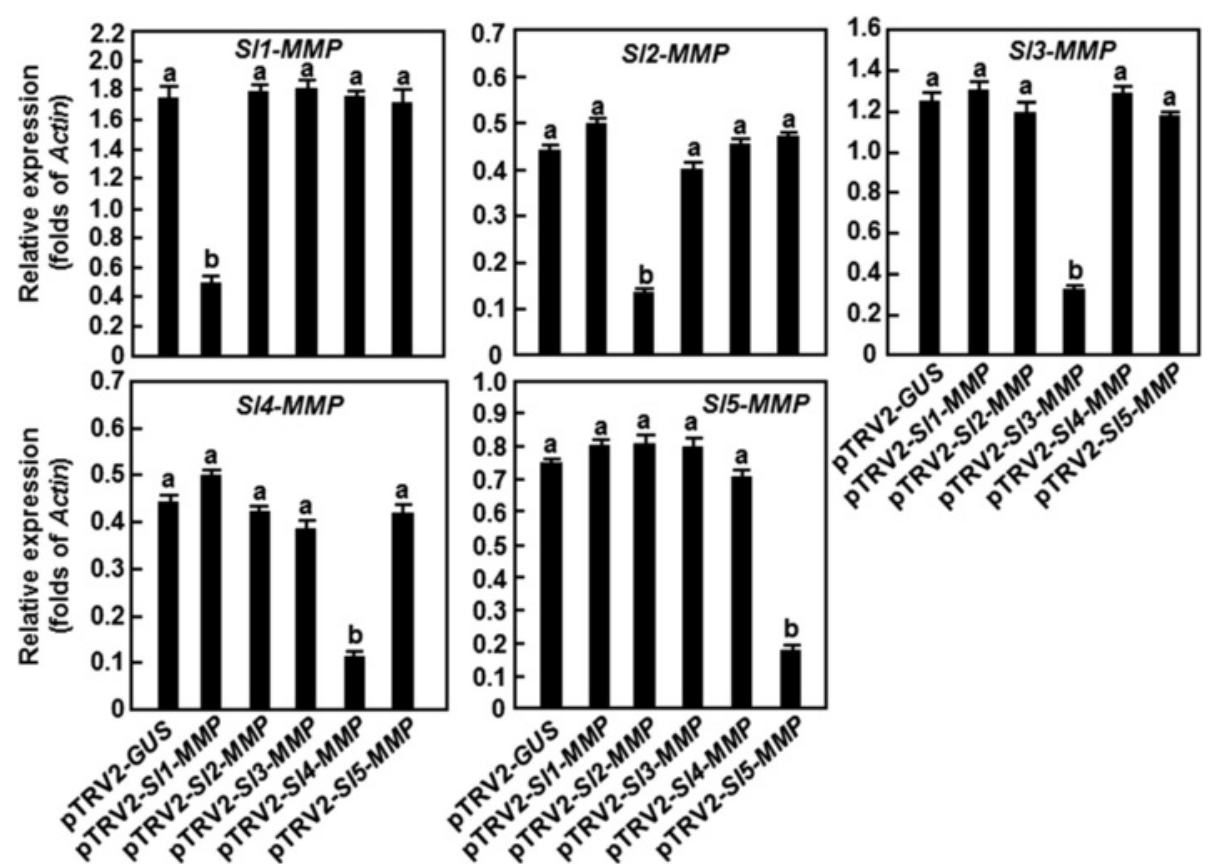

Fig. 4 Silencing efficiency and specificity for target genes in silenced plants. Two-week-old tomato seedlings were infiltrated with agrobacteria carrying PTRV2-SI-MMPs or PTRV2-GUS and leaf samples were collected at 4 weeks after agroinfiltration. Expression levels of each SI-MMP genes in targeted and nontargeted SI-MMP-silenced and non-silenced plants were analyzed by qRT-PCR and data obtained were normalized with actin transcript values. Data presented are the means \pm SD from three independent experiments and different letters above the columns indicate significant differences at $p<0.05$ level

pTRV2-GUS-infiltrated plants (Fig. 5a), at 3 days after inoculation (dpi), showing an approximately $60 \%$ of increase in lesion size over those on leaves from the pTRV2-GUS-infiltrated control plants (Fig. 5c). We further analyzed and compared the disease severity and in planta fungal growth in the pTRV2-Sl3-MMP- and pTRV2-GUS-infiltrated plants after inoculation by foliar spraying with spore suspension of $B$. cinerea in whole plant inoculation experiments. As shown in Fig. 5b, the pTRV2-GUS-infiltrated control plants displayed slight disease symptoms, whereas the pTRV2-Sl3-MMP-infiltrated plants showed severe diseases symptoms, showing large necrotic areas and maceration or wilting of full leaves at $5 \mathrm{dpi}$. Analysis of the transcript for the $B$. cinerea actin gene BcActin revealed that growth of $B$. cinerea in leaf tissues of the pTRV2-Sl3-MMP-infiltrated plants had 3 times higher than those in the pTRV2GUS-infiltrated control plants at 24 and $48 \mathrm{~h}$ after inoculation (Fig. 5d). These data indicate that silencing of the SI3-MMP resulted in reduced resistance to $B$. cinerea, demonstrating the requirement of Sl3-MMP for resistance to $B$. cinerea.

As mentioned above that silencing of Sl3-MMP resulted in a clear phenotype in change of disease resistance to $B$. cinerea, we subsequently focused our efforts on the functions in resistance to other diseases, mechanism and biochemical activity of Sl3-MMP. We examined whether $S l 3-M M P$ is also involved in resistance against Pst DC3000, which is a hemibiotrophic bacterial pathogen that has different infection style from that of $B$. cinerea. In our experiments, necrotic lesions were observed in the inoculated leaves of the pTRV2-Sl3-MMPand pTRV2-GUS- infiltrated plants; however, the lesions on leaves of the pTRV2-Sl3-MMP-infiltrated plants were larger and denser than those in the pTRV2-GUSinfiltrated plants (Fig. 6a). At 2 and 4 dpi, the bacterial population in the inoculated leaves of the pTRV2-Sl3MMP-infiltrated plants showed approximately 10 and 25 folds higher over those in the pTRV2-GUS-infiltrated plants, respectively (Fig. 6b). These results indicate that silencing of Sl3-MMP resulted in reduced resistance to Pst DC3000, implying the requirement of Sl3-MMP for resistance against Pst DC3000.

\section{Silencing of S/3-MMP attenuated defense response against $B$. cinerea}

To elucidate the possible mechanism involved in the reduced resistance in Sl3-MMP-silenced plants, we analyzed and compared the accumulation of reactive oxygen species (ROS), cell-death response and expression of defense genes before and after infection with $B$. cinerea between the Sl3-MMP-silenced plants and the control plants. No difference in accumulation of $\mathrm{H}_{2} \mathrm{O}_{2}$, as detected by 3, 3-diaminobenzidine (DAB) staining, was 


\section{(A)}

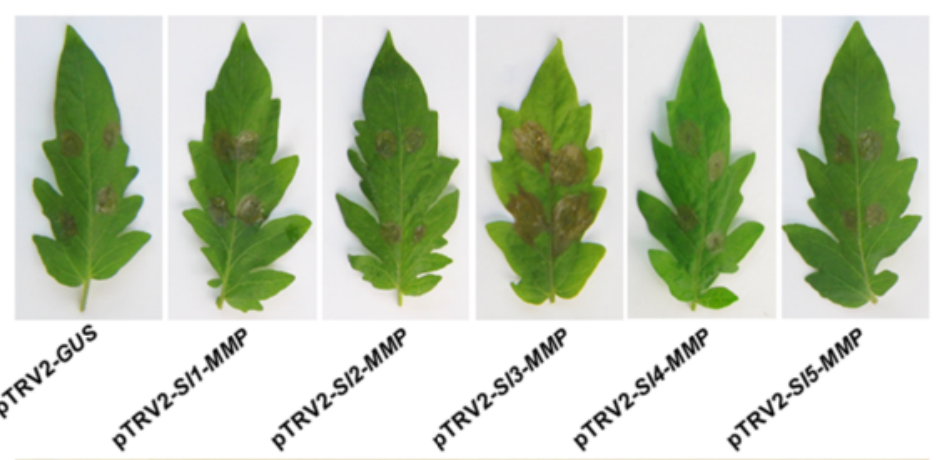

(B)
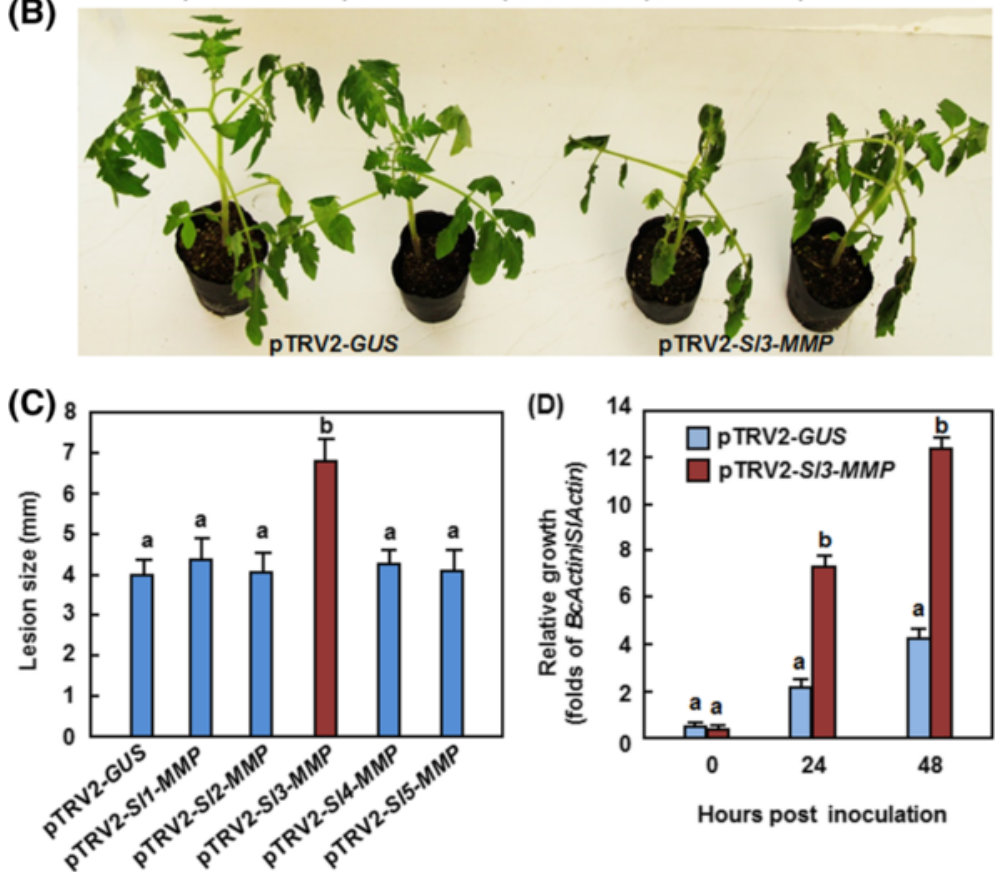

Fig. 5 Silencing of S/3-MMP resulted in reduced resistance to $B$. cinerea. Two-week-old seedlings were infiltrated with agrobacteria carrying pTRV2-SI-MMP or PTRV2-GUS and were inoculated at 4 weeks after VIGS infiltration by dropping spore suspension ( $1 \times 10^{5}$ spores $/ \mathrm{mL}$ ) on detached leaves or foliar spraying with spore suspension $\left(2 \times 10^{5}\right.$ spores $\left./ \mathrm{mL}\right)$ onto leaves of whole plants. a and $\mathbf{b}$ Disease phenotype and lesion sizes in leaves of the pTRV2-SI-MMPs- and pTRV2-GUS-infiltrated plants in detached leaf inoculation assays. Lesion sizes were measured at 3 days after inoculation on a minimum of 20 leaves in each experiment. $\mathbf{c}$ and $\mathbf{d}$ Disease phenotype on and fungal growth in the pTRV2-SI3-MMP- and pTRV2GUS-infiltrated plants in whole plant inoculation assays. Fungal growth in planta was estimated by analyzing the transcript levels of BcActin gene by qRTPCR using SIActin as an internal control at the indicated time points after inoculation. Data presented in $\mathbf{b}$ and $\mathbf{d}$ are the means \pm SD from three independent experiments and different letters above the columns indicate significant differences at $p<0.05$ level

observed in leaves of pTRV2-Sl3-MMP- and pTRV2GUS-infiltrated plants without infection of $B$. cinerea (Fig. 7a), indicating that silencing of Sl3-MMP itself did not affect the generation and accumulation of $\mathrm{H}_{2} \mathrm{O}_{2}$ in tomato plants. After infection with $B$. cinerea, significant accumulation of $\mathrm{H}_{2} \mathrm{O}_{2}$, shown as brown precipitates in leaves, was detected in leaves of pTRV2-Sl3-MMP- and pTRV2-GUS-infiltrated plants (Fig. 7a). However, the leaves from pTRV2-Sl3-MMP-infiltrated plants showed consistent increase in intensity of the stained areas (Fig. 7a), showing increases of $85 \%$ at $12 \mathrm{~h}$ and $24 \% 24 \mathrm{~h}$, when compared with those in pTRV2-GUS-infiltrated plants after infection of $B$. cinerea (Fig. 7b). On the other hand, levels of cell death, as detected by Trypan blue staining, and electrolyte leakage, as estimated by ion conductivity, were comparable in leaves of pTRV2-Sl3-MMPand pTRV2-GUS-infiltrated plants without infection of $B$. cinerea but significantly increased after infection with $B$. cinerea (Fig. 7c and d). Notably, the levels of cell death and electrolyte leakage in leaves of pTRV2-Sl3-MMP-infiltrated plants were significantly higher than those in leaves of pTRV2-GUS-infiltrated plants after infection of $B$. cinerea (Fig. 7c), leading to $38 \%$ increases for electrolyte leakage at $24 \mathrm{~h}$ after infection (Fig. 7d). These data 

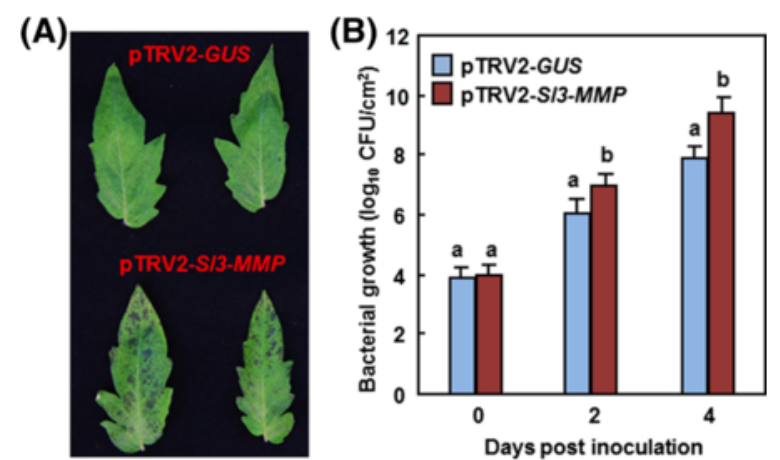

Fig. 6 Silencing of S/3-MMP resulted in reduced resistance to $P$. syringae pv. tomato DC3000. Two-week-old seedlings were infiltrated with agrobacteria carrying PTRV2-SI3-MMP or PTRV2-GUS and were inoculated by infiltration with Pst DC3000 4 weeks after VIGS infiltration. a Representative disease phenotype. b Bacterial population. Data presented in $\mathbf{b}$ are the means \pm SD from three independent experiments and different letters above the columns indicate significant differences at $p<0.05$ level indicate that silencing of Sl3-MMP resulted in increased ROS accumulation of $\mathrm{H}_{2} \mathrm{O}_{2}$ and excessive cell death in pTRV2-Sl3-MMP-infiltrated plants upon infection of $B$. cinerea.

To explore the possible mechanism for the increased accumulation of $\mathrm{H}_{2} \mathrm{O}_{2}$ in the Sl3-MMP-silenced plants, we analyzed and compared the expression of genes encoding for NADPH oxidases, glutathione reductase (GR), catalases (CAT), superoxide dismutases (SOD) and ascorbate peroxidases (APX) in the pTRV2-Sl3-MMPinfiltrated plants. As shown in Fig. 8a, no significant difference in the expression levels of these selected genes was observed between the pTRV2-GUS-infiltrated and pTRV2-Sl3-MMP-infiltrated plants without infection of $B$. cinerea. By contrast, the expression levels of Rboh1 and Wfi1, two genes for NADPH oxidases, in the pTRV2-Sl3-MMP-infiltrated plants were significantly elevated upon Botrytis infection, showing $\sim 5$-fold increases over those in the pTRV2-GUS-infiltrated plants. Similarly, the expression levels of $A P X$ and $G R$ in the pTRV2-Sl3-MMP-infiltrated plants were also increased as compared with those in the pTRV2-GUS-infiltrated plants (Fig. 8a). By contrast, no significant difference
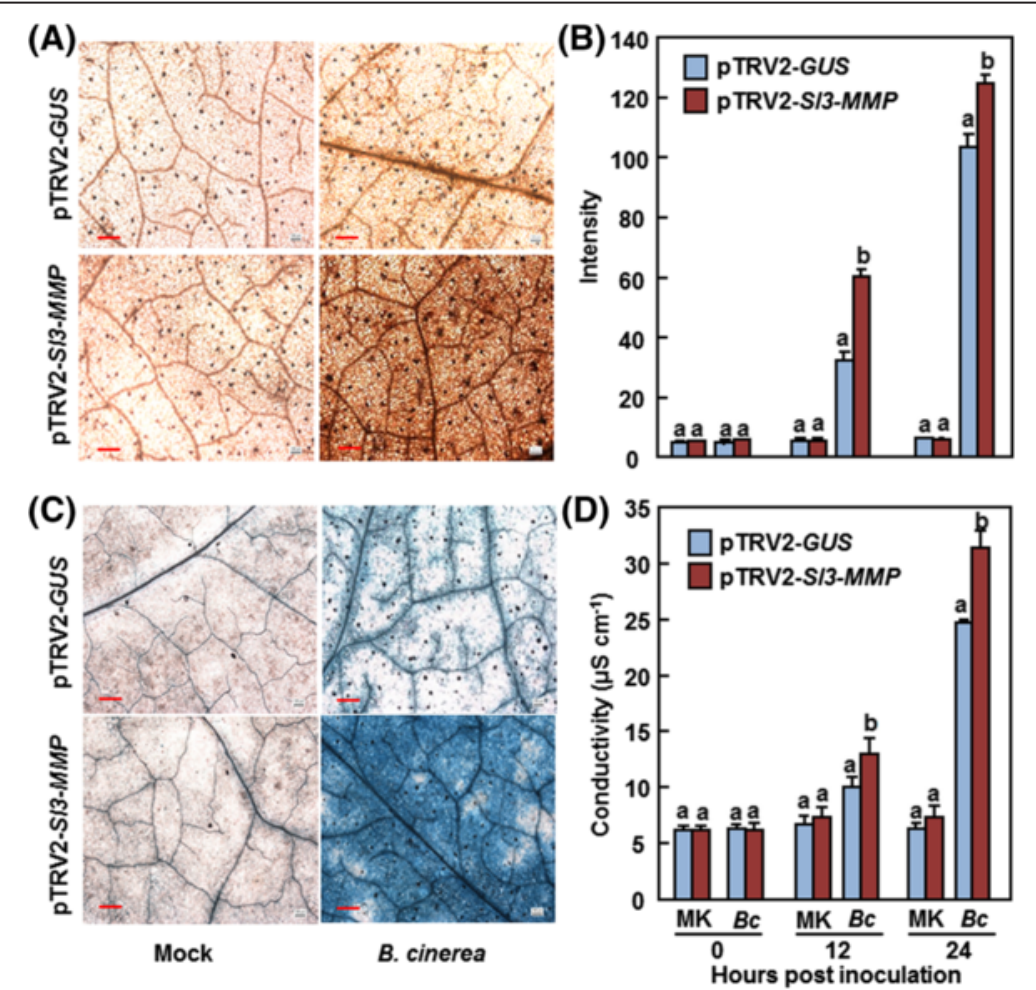

Fig. 7 Increased accumulation of $\mathrm{H}_{2} \mathrm{O}_{2}$ and cell death in SI3-MMP-silenced plants after infection with $B$. cinerea. Two-week-old seedlings were infiltrated with agrobacteria carrying pTRV2-SI3-MMP or pTRV2-GUS and were inoculated with spore suspension of B. cinerea or with buffer solution as a mockinoculation control at 4 weeks after VIGS infiltration. Leaves from six individual plants were collected at $24 \mathrm{~h}$ after inoculation and used for analysis of cell death and $\mathrm{H}_{2} \mathrm{O}_{2}$ accumulation. $\mathbf{a}$ and $\mathbf{b}$ Accumulation of $\mathrm{H}_{2} \mathrm{O}_{2}$ by DAB staining and quantification method respectively. c Cell death detected by trypan blue staining. $\mathbf{d}$ Electrolyte leakage. Data presented in $\mathbf{b}$ and $\mathbf{d}$ are the means \pm SD from three independent experiments and different letters above the columns indicate significant differences at $p<0.05$ level 


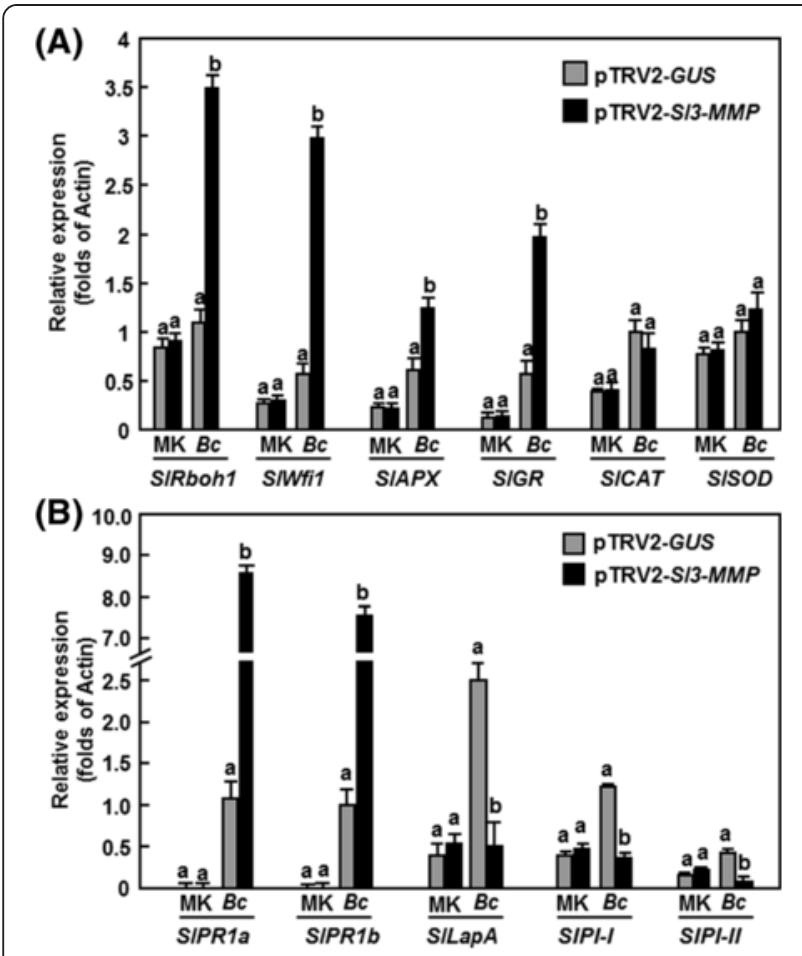

Fig. 8 Silencing of S/3-MMP affected the expression of ROS generation- and scavenging-related genes and defense-mediated genes after infection of $B$. cinerea. Two-week-old seedlings were infiltrated with agrobacteria carrying pTRV2-SI3-MMP or PTRV2-GUS and were inoculated with spore suspension of $B$. cinerea or with buffer as a mock-inoculation control at 4 weeks after VIGS infiltration. Leaves from six individual plants were collected at $24 \mathrm{~h}$ after inoculation and used for analysis of gene expression. a Expression of ROS generationand scavenging-related genes. $\mathbf{b}$ Expression of defense-related genes. Relative expression levels were shown as folds of the actin transcript values. Data presented are the means \pm SD from three independent experiments and different letters above the columns indicate significant differences at $p<0.05$ level. MK, mock-inoculated control; $\mathrm{BC}, B$. cinerea-inoculated treatment

was observed in the expression levels of $C A T$ and $S O D$ between the pTRV2-Sl3-MMP-infiltrated plants and the pTRV2-GUS-infiltrated plants (Fig. 8a). These results indicate that the increased ROS accumulation in the Sl3MMP-silenced plants might be due to an increased ROS generating ability resulted from the high level of expression of the NADPH oxidases.

We next analyzed the expression changes of defenserelated genes regulated by the JA/ET- and SA-mediated signaling pathways, respectively, to explore the possible molecular mechanism associated with the reduced disease resistance in Sl3-MMP-silenced plants. No significant difference in expression of SlPR1a and SlPR $1 b$, known to be regulated by the SA-mediated signaling pathway [27], and SlLapA, SIPI-I and SlPI-II, known to be regulated by the JA/ET-mediated signaling pathway [27], was observed in mock-inoculated pTRV2-Sl3-
MMP- or pTRV2-GUS-infiltrated plants (Fig. 8b), indicating that silencing of Sl3-MMP did not affect the expression of defense-related genes in tomato plants. However, the expression of these defense-related genes exhibited different patterns in pTRV2-Sl3-MMP- or pTRV2-GUS-infiltrated plants after infection of $B$. cinerea (Fig. 8b). The expression of SIPR1a and SlPR1b in the pTRV2-Sl3-MMP- and pTRV2-GUS-infiltrated plants was significantly upregulated after infection of $B$. cinerea, but the levels in pTRV2-Sl3-MMP-infiltrated plants showed 7-8 folds higher than those in the pTRV2-GUS-infiltrated plants (Fig. 8b). After infection with B. cinerea, the expression levels of SlLapA, SIPI-I and SIPI-II in pTRV2-GUS-infiltrated plants were significantly increased, whereas the levels in pTRV2-Sl3MMP-infiltrated plants remained unchanged, comparable to those in the mock-inoculated plants but showing 3-5 folds of decreases as compared with those in $B$. cinerea-infected pTRV2-GUS-infiltrated plants (Fig. 8b). These data demonstrate that silencing of Sl3-MMP attenuated the defense response in tomato upon infection of $B$. cinerea through affecting the expression of defense-related genes that are regulated by the JA/ETmediated signaling pathway.

\section{Transient expression of S/3-MMP in Nicotiana}

benthamiana led to increased resistance against $B$. cinerea To further confirm the function of Sl3-MMP in disease resistance, we examined whether overexpression of Sl3$M M P$ could confer an increased resistance to B. cinerea. In our qRT-PCR experiments, transcripts of putative $N$. benthamiana homolog(s) of Sl3-MMP was detected using Sl3-MMP-specific primers in GFP-infiltrated plants, probably due to high level of sequence similarity/identity among Sl3-MMP and the homologous $M M P$ genes in $N$. benthamiana. However, agroinfiltration did not significantly affect the transcript levels of endogenous $N$. benthamiana homologous genes in GFP-infiltrated plants (Fig. 9a). When transiently expressed in $N$. benthamiana leaves, high levels of Sl3-MMP expression, as estimated by the significant increases in the transcript levels of Sl3$M M P$ and the endogenous homologous genes in Sl3MMP-infiltrated plants over the levels of the endogenous homologous genes in GFP-infiltrated plants, and the Sl3MMP-GFP (a fusion of Sl3-MMP with GFP) fusion protein were detected during a period of $48 \mathrm{~h}$ after infiltration (Fig. 9a and b). In disease assays, the lesions on leaves from Sl3-MMP-infiltrated $N$. benthamiana plants were significantly smaller than that in GFP-infiltrated control plants (Fig. 9c), leading to approximately $40 \%$ of reduction in lesion size at 5 days after inoculation (Fig. 9d). To examine whether an increased defense response was linked to the enhanced resistance resulted from the transient expression of $S l 3-M M P$, we analyzed and compared 


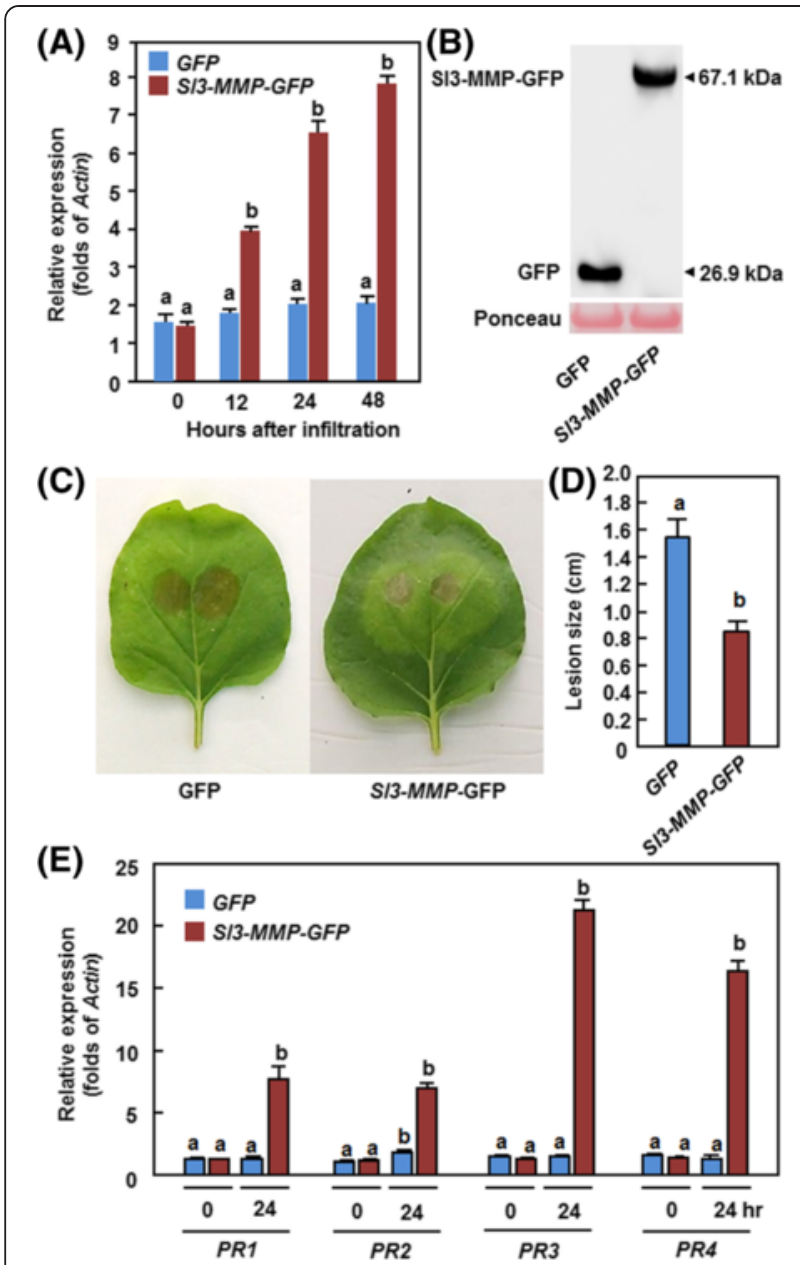

Fig. 9 Transient expression of S/3-MMP in N. benthamiana conferred an increased resistance to $B$. cinerea. a Expression of SI3-MMP. Agrobacteria carrying pFGC-SI3-MMP or pFGC-eGFP were infiltrated into leaves of $N$. benthamiana and expression of S/3-MMP was analyzed by qRT-PCR. Relative expression levels were calculated by comparing with the corresponding values at $0 \mathrm{~h}$ (as a control) after infiltration. b Immunoblot analysis of SI3-MMP-GFP fusion proteins in N. benthamiana leaves at $48 \mathrm{~h}$ after agroinfiltration. A GFP-specific antibody was used for detection of GFP-fusion protein. Equal loading of total proteins was examined by Ponceau staining. $\mathbf{c}$ and $\mathbf{d}$ Disease symptom and lesion size. e Expression of defense-related genes. Opposite part of the leaves infiltrated with SI3-MMP-GFP or pFGC-eGFP was inoculated by dropping spore suspension $\left(2 \times 10^{5}\right.$ spores $\left./ \mathrm{mL}\right)$ of $B$. cinerea and lesion sizes were measured at 5 days after inoculation. Data presented in $\mathbf{d}$ are the means \pm SD from a minimum of 60 lesions. Data presented in $\mathbf{a}$ and $\mathbf{e}$ are the means \pm SD from three independent experiments and different letters above the columns indicate significant differences at $p<0.05$ level

the expression of some selected defense-related genes in leaves of the GFP- and Sl3-MMP-infiltrated N. benthamiana plants. As shown in Fig. 9e, the expression of $P R 1$, PR2, PR3 and PR4 in S13-MMP-infiltrated plants were significantly upregulated at $24 \mathrm{~h}$ after infiltration, showing 5 24 folds of increases over those in GFP-infiltrated plants, whereas no significant difference in the levels of these defense-related genes was observed between GFP-infiltrated
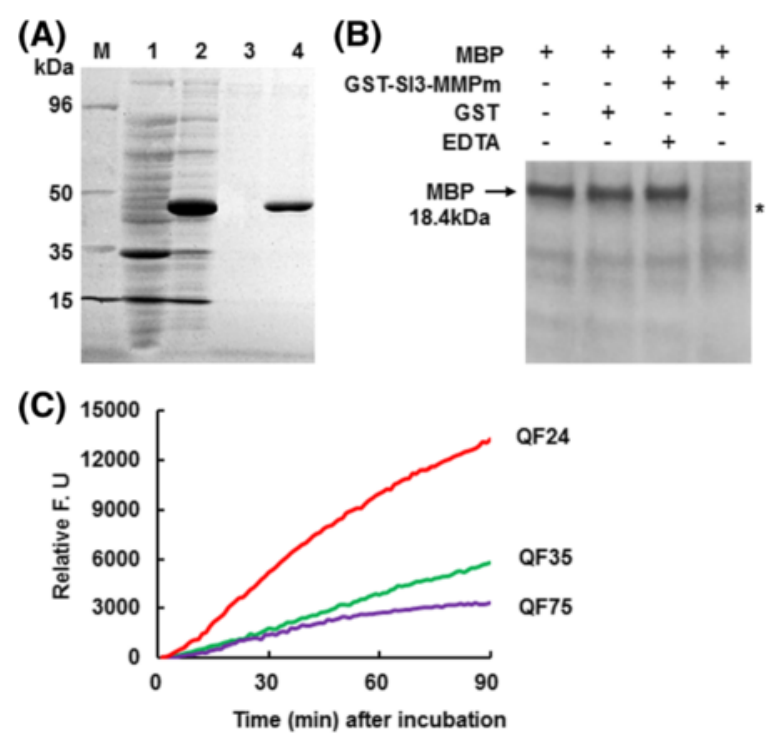

Fig. 10 Mature SI3-MMP protein had proteolytic activity with different cleavage site specificity. a Purification of recombinant SI3-MMPm. M, marker for protein molecular weight; lane 1, total cell proteins from non-induced bacteria carrying PGEX-SI3-MMPm; lane 2, total cell protein from induced bacteria carrying pGEX-SI3-MMPm; lane 3, purified protein from non-induced bacteria carrying PGEX-SI3-MMPm; lane 4, purified SI3-MMP protein. b Proteolytic activity of SI3-MMPm on MBP in the absence (-) or presence (+) of 1 mM EDTA. Reactions with different component combinations were incubated with $5 \mu \mathrm{g}$ of MBP at $37^{\circ} \mathrm{C}$ and the products were resolved by $16 \%$ SDS-PAGE. Lane 1 , a negative control containing MBP only; Lane 2, a negative control with GST tag; Lane 3, a reaction containing SI3-MMPm and 1 mM EDTA; lane 4 , a reaction containing SI3-MMPm. * indicates the product degraded from MBP by SI3-MMPm. c Cleavage site specificity of SI3MMPm on synthetic quenched peptide substrates. Enzymatic activity of SI3-MMPm on synthetic quenched peptides QF24, QF35 and QF75 was measured as relative fluorescence in arbitrary units over $90 \mathrm{~min}$ at 1 min intervals. Values are the means of triplicate measurements

plants at $0 \mathrm{~h}$ and $24 \mathrm{~h}$ and between GFP- and Sl3-MMPinfiltrated plants at $0 \mathrm{~h}$ after infiltration (Fig. 9e). These data demonstrate that transient expression of Sl3-MMP in $N$. benthamiana plants conferred an increased resistance against $B$. cinerea through an activated defense response resulted from the upregulated expression of defenserelated genes.

\section{Proteolytic activity and subcellular localization of SI3-MMP}

To delineate the biochemical activity of Sl3-MMP, we expressed the putative mature form of S13-MMP $\left(\right.$ Gly $\left.^{155}-\mathrm{Ser}^{340}\right)$, in which the $\mathrm{N}$-terminal propeptide domain as well as the C-terminal predicted transmembrane domains were deleted, in E. coli, and purified the recombinant mature Sl3-MMP protein (Sl3-MMPm) to homogeneity as examined on a SDS-PAGE gel (Fig. 10a). The activity of the recombinant SI3-MMPm was examined for its ability to cleave a general protease substrate bovine myelin basic protein (MBP). As shown in Fig. 10b, 
MBP was present as a single band without any degradation in absence of Sl3-MMPm and the GST tag alone did not cause degradation of MBP. However, significant degradation of MBP was detected in the presence of Sl3-MMPm, showing an additional band with small molecular weight (Fig. 10b). Meanwhile, addition of $1 \mathrm{mM}$ EDTA, an inhibitor of MMP [2], in the Sl3-MMPm-MBP reaction completely abolished the degradation of MBP by Sl3-MMPm (Fig. 10b). These results demonstrate that the recombinant Sl3-MMPm had proteolytic activity on MBP. To determine the cleavage site specificity, we examined whether Sl3-MMPm was able to hydrolyze quenched fluorescent (QF) peptide substrates QF24, QF35 and QF75. As shown in Fig. 10c, the recombinant Sl3-MMPm cleaved the QF24 most efficiently, followed by QF 35 and then QF75, indicating a cleavage site specificity of Sl3MMP towards different substrates.

Subcellular localization of Sl3-MMP was examined by transient expression of a GFP-tagged Sl3-MMP construct in onion epidermal cells. To exclude the possibility that Sl3-MMP-GFP is secreted into the apoplast and not associated with the plasma membrane, the GFPtagged Sl3-MMP construct was introduced into onion epidermal cells by particle bombardment and GFP was observed before and after plasmolysis, which was induced by rinsing the cells with $0.8 \mathrm{M}$ mannitol solution for $10 \mathrm{~min}$. The GFP protein alone accumulated in the cells without specific localization; however, GFP from the GFP-tagged Sl3-MMP protein was clearly localized on the plasma membrane before and after plasmolysis and no GFP was detected in the space between plasma membrane and cell wall (Fig. 11). These observations indicate that Sl3-MMP was targeted to plasma membrane of cells but not secreted into the apoplast.

\section{Discussion}

MMPs are a family of zinc-dependent endopeptidases widely distributed in all organisms. However, only a few of plant MMPs have been studied for their biological functions so far [2]. In the present study, we characterized the tomato MMP family, analyzed the expression patterns of Sl-MMP genes in response to pathogen infection and treatments of defense-related signaling hormones, and performed VIGS- and transient expression-based functional analyses to explore the involvement of Sl-MMPs in disease resistance. Our results demonstrate that Sl3-MMP act as a positive regulator of defense response against $B$. cinerea and Pst DC3000 in tomato, providing new insights into the biological function of plant MMPs.

The significance of the proteases is generally related to their substrates and the physiological consequences of these actions. No physiological substrate has been identified for higher plant MMPs. However, activity of plant

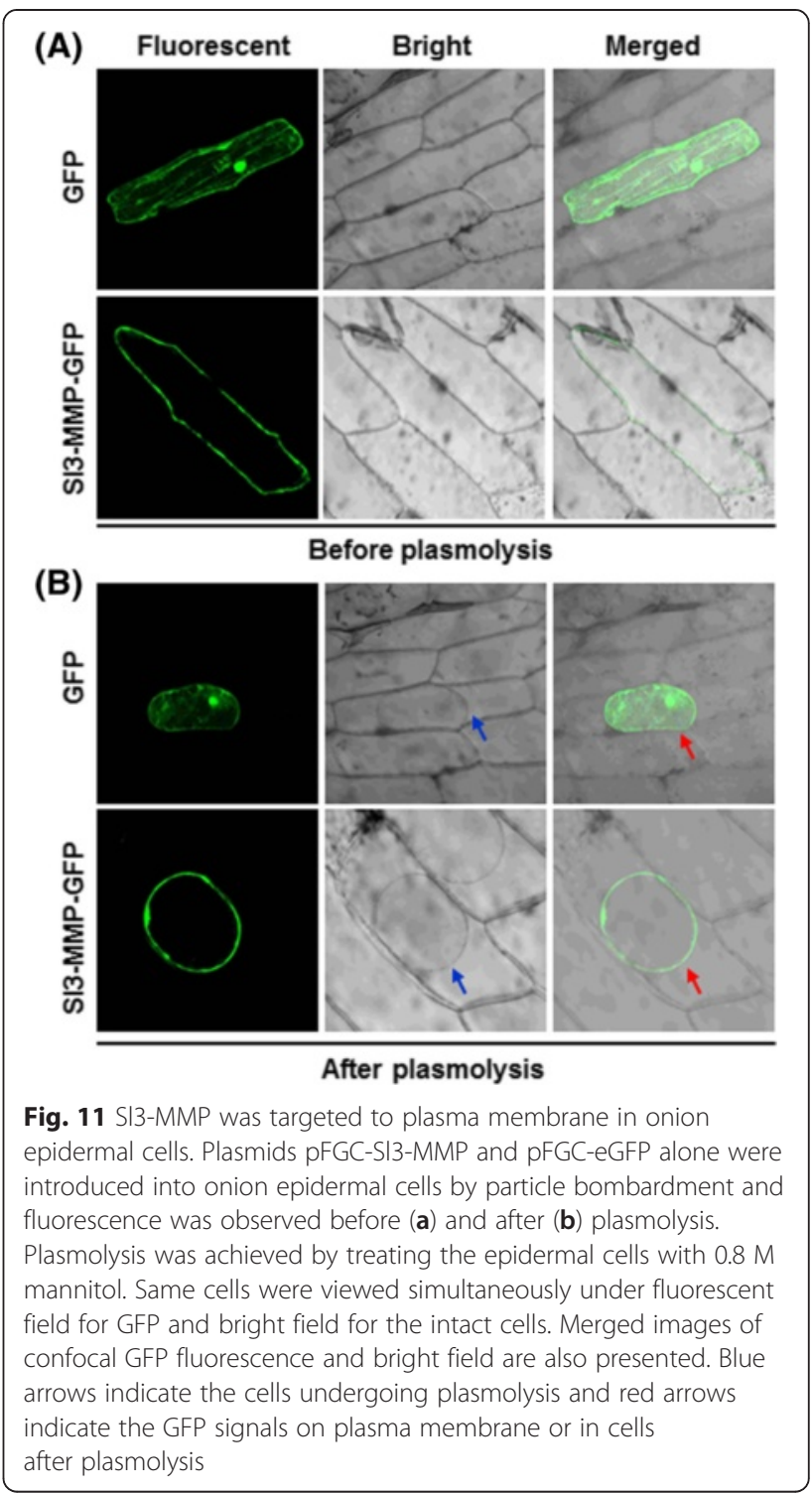

MMPs was detected in vitro on general animal protease substrates [11, 12, 15, 17, 19, 28, 29]. It was also found that recombinant mature MMPs, lacking the $\mathrm{N}$-terminal propeptide domains showed higher proteolytic activity than the full-length MMPs $[15,19,28]$. In the present study, we demonstrated that Sl3-MMPm, lacking the Nterminal propeptide domain, had proteolytic activity toward MBP in vitro (Fig. 10b). Similar proteolytic activity on MBP was verified for At1-MMP, At2-MMP, At5MMP [15, 30], Gm2-MMP [19] and Pta1-MMP [12]. However, proteolytic activities of plant MMPs on other substrates were also detected, e.g., Cs1-MMPand Nt1MMP on gelatin $[17,28]$ and At3-MMP and Nt1-MMP on $\beta$-casein $[11,28,30]$. Therefore, it is likely that members of plant MMP family may have different substrates in plants and such substrate specificity may determine their biological functions. On the other hand, previous 
reports have shown that both human and plant MMPs differ in their cleavage site preferences [30]. Synthetic peptides, designed for vertebrate MMPs, have been used to characterize the proteolytic activity of SMEP1/Gm1MMP [29], At1-MMP-At5-MMP [15, 30], Cs1-MMP [17] and Nt1-MMP [28]. Among these synthetic peptides, QF24 is a substrate for all MMPs [31], QF35 is the stromelysin substrate [32] and QF75 was designed to mimic the activation cleavage site in human MMP-2 and has an amino acid sequence different from the typical MMP cleavage motif [30]. In the present study, we found that Sl3-MMPm, lacking the N-terminal propeptide domain, cleaved the QF24 with the highest efficiency, followed by QF35 and QF75 (Fig. 10c). Thus, Sl3-MMP is able to efficiently cleave general MMP substrates. This is in agreement with the differences in the cleavage site specificities observed in Arabidopsis MMPs [30]. For example, At1-MMP was found to cleave efficiently QF24 and QF35 while only At5-MMP was found to be able to cleave QF75 $[15,30]$.

Most human MMPs are secreted into the extracellular matrix, but six membrane-type MMPs maintain contact with the cell surface through either transmembrane domains or glycosylphosphatidylinositol (GPI) anchors [33]. Like some plant MMPs such as GmMMP2, At2MMP, At4-MMP and At5-MMP [20], Sl3-MMP contains a signal peptide (1-21 aa) at the $\mathrm{N}$-terminus and a putative GPI-anchor modification site or a transmembrane domain at the $\mathrm{C}$-terminus and was predicted to be located in the extracellular space by WoLF PSORT and TargetP programs. Our experimental evidence from transient expression of the GFP-tagged Sl3-MMP in onion epidermal cells clearly demonstrated that Sl3MMP is targeted to plasma membrane but not secreted into the apoplast (Fig. 11). This is consistent with the previous observations that both of soybean Slti114 and tobacco Nt1-MMP, which are phylogenetically closest MMPs to Sl3-MMP, localized in the plasma membrane $[11,34]$, but differs from the soybean SMEP1, which was reported to be tightly bound to the cell wall [21]. Animal MMPs have been recognized as a class of enzymes that plays a critical role in ECM turnover and remodeling based on their ability to hydrolyze the major protein components of the ECM [35]. However, whether Sl3-MMP exerts its biological function through degradation of plant ECM components needs to be further investigated.

Direct evidence supporting the involvement of MMPs in plant disease resistance is lacking although members of the MMP family have been shown to be induced by pathogen infection and defense signaling hormones $[2,20]$. In the present study, we observed that the expression of Sl-MMPs could be induced by both Pst DC3000 and B. cinerea (Figs. 3 and 4). This is similar to the observations that the expression of Gm2-MMP in soybean
[19] and Nt1-MMP in tobacco suspension cells [11] was induced by different fungal, oomycetes or bacterial pathogens. However, pathogen-induced expression patterns varied among the $S l-M M P s$, e.g., all Sl-MMPs, especially for Sl1-MMP, Sl3-MMP and Sl5-MMP, exhibited upregulated expression patterns in response to $B$. cinerea while only Sl1-MMP, Sl2-MMP and Sl3-MMP showed upregulated patterns in response to Pst DC3000 (Fig. 2). In addition, the expression of most of the Sl-MMPs was also induced by defense signaling hormones such as SA, JA and ACC (Fig. 3). This is consistent with At2-MMP whose expression was induced rapidly by MeJA [18] but differs from Gm2-MMP, which was not induced by SA and MeJA in soybean suspension cells [19]. Therefore, it is likely that the expression of Sl-MMPs is precisely controlled by complex mechanisms in response to infection from different pathogens and/or defense signaling hormones.

Our VIGS- and transient expression-based functional analyses led to the identification of Sl3-MMP as a positive regulator of defense response against $B$. cinerea. Firstly, silencing of Sl3-MMP but not other four Sl$M M P S$ resulted in reduced resistance against $B$. cinerea and Pst DC3000, as the Sl3-MMP-silenced plants exhibited severe disease symptoms and supported more in planta pathogen growth (Figs. 6 and 7). Accompanying with the reduced resistance in Sl3-MMP-silenced plants was attenuated defense responses upon infection of $B$. cinerea, e.g., increased ROS accumulation and cell death and downregulated expression of JA/ET-mediated signaling responsive defense-related genes (Figs. 8 and 9). B. cinerea can induce the generation of ROS in plants to benefit its infection [36-38]; however, ROS may function in different ways in the interaction between tomato and B. cinerea [39]. It is generally accepted that ROS accumulated during the late stage directly benefits the establishment of infection by B. cinerea [39] and that sustained production of ROS as a facilitator of cell death may promote susceptibility [40]. In the present study, the Sl3-MMP-silenced plants accumulated larger amount of $\mathrm{H}_{2} \mathrm{O}_{2}$ at $24 \mathrm{~h}$ than the control plants after inoculation (Fig. 7a and b). This increase in ROS accumulation in the Sl3-MMP-silenced plants was mainly due to an accelerated ROS generation rather than a reduced ROS scavenging ability because the expression levels of SlRboh1 and SlWfil, coding for NADPH oxidases that are plasma membrane-localized ROS generating enzymes [41], were significantly higher but the expression levels of SlCAT and SISOD, coding enzymes capable of scavenging ROS [42] were similar to those in the control plants after infection of B. cinerea (Fig. 8a). Increased expression levels of SlAPX and SlGR in the Sl3-MMP-silenced plants might be responses to the change in cellular redox status caused by the excessive ROS accumulated after infection of $B$. 
cinerea. Furthermore, excessive ROS accumulation in the Sl3-MMP-silenced plants thus resulted in an increased level of cell death, as revealed by Trypan blue staining and measurement of electrolyte leakage (Fig. 7c and d), which should favor the growth of B. cinerea (Fig. 5d). It is therefore likely that accelerated ROS accumulation in B. cinerea-infected Sl3-MMP-silenced plants may be one of the mechanisms leading to a reduced resistance to $B$. cinerea. On the other hand, B. cinerea-induced expressions of SIPR1a and $S I P R 1 b$, regulated by the SA-mediated signaling pathway [27], and SILapA, SIPI-I, and SIPI-II, regulated by the JA/ ET-mediated signaling pathway [27], were significantly increased and suppressed, respectively, in Sl3-MMP-silenced plants (Fig. 9b), indicating that silencing of $S l 3-M M P$ may affect the efficiency of the JA/ET-mediated signaling pathway in regulating expression of defense-related genes. Secondly, transient expression of Sl3-MMP in $N$. benthamiana plants conferred an enhanced resistance to $B$. cinerea and upregulated expression of defense genes (Fig. 9), further supporting the hypothesis that Sl3-MMP acts as a positive regulator of defense response against $B$. cinerea. This is consistent with the observations that ectopic overexpression of MtMMPL1 led to numerous abortive infections and an overall decrease in the number of nodules upon Sinorhizobium meliloti infection [13]. Additionally, silencing of Sl3-MMP also led to a reduced resistance to Pst DC3000, a hemibiotrophic bacterial pathogen that has different life style from that of $B$. cinerea, indicating a broad involvement of Sl3-MMP in regulation of disease resistance against different pathogens.

\section{Conclusion}

Tomato genome encodes five Sl-MMP genes and all of them exhibited differentially expression patterns in response to pathogens and defense signaling hormones. The present study focused on the characterization of SlMMPs in disease resistance and data from VIGS- and transient expression-based analyses demonstrated that Sl3-MMP functions as a positive regulator of defense response against B. cinerea and Pst DC3000 in tomato. Further biochemical studies indicate that Sl3-MMP possess an in vitro enzymatic activity with different cleavage site specificities and is targeted on the plasma membrane. However, the biological functions of other four Sl-MMPs need to be investigated. Further identification of endogenous substrates for Sl3-MMPs will be helpful in elucidation of the biochemical mechanism of Sl3MMP in disease resistance.

\section{Methods}

\section{Plant growth, treatment and disease assays}

Tomato (Solanum lycopersicum L.) cv. Suhong 2003 was used for all experiments. Plants were grown in a mixture of perlite: vermiculite: plant ash (1:6:2) in a growth room under fluorescent light $\left(200 \mu \mathrm{E} \mathrm{m} \mathrm{m}^{2}\right)$ at $22-24{ }^{\circ} \mathrm{C}$ with $60 \%$ relative humidity and a $14 \mathrm{~h}$ light/10 h dark cycle. Pathogen inoculation, disease assays and measurement of pathogen in planta growth were performed basically according to previously described protocols $[43,44]$. For analysis of gene expression in responding to pathogen infection, mock-inoculation controls were set by treating the plants with buffer solution (B. cinerea) or $10 \mathrm{mM}$ $\mathrm{MgCl}_{2}$ (Pst DC3000). For analysis of gene expression in response to defense signaling hormones, 4-week-old tomato plants were treated by foliar spraying with $100 \mu \mathrm{M}$ MeJA, $100 \mu \mathrm{M}$ ACC or $100 \mu \mathrm{M}$ SA in $0.1 \%$ ethanol and equal volume of $0.1 \%$ ethanol solution as control. Leaf samples were collected at indicated time points after treatment or inoculation and stored at $-80{ }^{\circ} \mathrm{C}$ until use.

\section{Identification of tomato SI-MMPs and bioinformatics analyses}

Arabidopsis AtMMPs were used as queries to perform multiple database searches against the proteome and genome files downloaded from the SOL Genomics Network (SGN, http://solgenomics.net) [45]. BlastP and TBlastN at NCBI (http://blast.ncbi.nlm.nih.gov) were performed with an e-value cutoff set to $1 \mathrm{e}-003$ [46]. All protein sequences were compared with known MMP sequences using ClustalX (http://www.clustal.org/) to verify the sequences were candidate MMPs. The obtained MMP sequences were examined by domain analysis programs PFAM (http://pfam.sanger.ac.uk/) and SMART (http:// smart.embl-heidelberg.de/) with the default cutoff parameters [47, 48]. The isoelectric points and molecular weights were predicted with the help of the proteomics and sequence analysis tools on the ExPASy Proteomics Server (http://expasy.org/). Sequence alignment was carried out by the ClustalX program [49]. Putative signal peptides and transmembrane domains were predicted by SignalP 4.1 (http://www.cbs.dtu.dk/services/SignalP/) and TMpred (http://ch.embnet.org/software/TMPRED_form. html), respectively. Phylogenetic trees including the tomato MMP protein sequences were constructed using the neighbor-joining (NJ) method of the MEGA6 program with the p-distance and complete deletion option parameters [50]. The reliability of the obtained trees was tested using a bootstrapping method with 1000 replicates.

\section{Cloning of the SI-MMP genes}

The coding sequence of the Sl3-MMP gene was amplified using a pair of gene-specific primers (Additional file 3) from tomato cDNAs and cloned into pMD19-T vector, yielding plasmids pMD19-Sl-MMPs. After confirmation by sequencing, the plasmid pMD19-Sl3-MMP was used for further experiments. 


\section{Purification of recombinant SI3-MMP protein and proteolytic activity assays}

The coding sequence for the mature form of Sl3-MMP protein (S13-MMPm) was amplified from pMD19-S13MMP with a pair of primers (Additional file 3) and cloned into pGEX-4 T-3 vector at EcoRI/XhoI sites, which was fused to glutathione-S-tranferase (GST) at its $\mathrm{N}$-terminal. The recombinant plasmid pGEX-Sl3MMPm and empty vector were introduced into the $E$. coli strain Rosetta DE3 and expression of S13-MMPm fusion and GST tag in E. coli cells was induced by $1 \mathrm{mM}$ isopropyl-D-thiogalactoside (IPTG) at $20{ }^{\circ} \mathrm{C}$ overnight. The GST-tagged Sl3-MMPm fusion protein and GST tag were purified using the Bug-Buster GST-Bind purification kit following the manufacturer's protocols (Merck, Darmstadt, Germany). Protein concentration was determined using Bio-Rad protein assay kit (BioRad, CA, USA) following the recommended method.

For proteolytic activity assays, purified Sl3-MMPm or GST (a negative control) was incubated with $5 \mu \mathrm{g}$ of MBP (Sigma-Aldrich, St. Louis, MO, USA) in the absence (-) or presence (+) of $1 \mathrm{mM}$ EDTA in $200 \mathrm{mM}$ Tris- $\mathrm{HCl}$, pH7.5, containing $10 \mathrm{mM} \mathrm{CaCl}_{2}, 0.1 \%$ (w/v) Brij35 and $1 \times$ EDTA-free protease inhibitor cocktail (Roche Diagnostics, Mannheim, Germany) at $37^{\circ} \mathrm{C}$ overnight. Products were analyzed by $16 \%$ Tricine-SDSPAGE and stained in Colloidal Coomassie [51].

Proteolytic activity of SI3-MMP were measured using the synthetic fluorescent substrates Mca-Pro-Leu-Gly-LeuDpa-Ala-Arg-NH2 (QF-24), Mca-Pro-Leu-Ala-Nva-DpaAla-Arg-NH2 (QF-35) and Mca-KESAbuNLFVLKDpaR-N H2 (QF-75) (synthesized by Invitrogen Life Technologies, Inc.). Purified recombinant Sl3-MMPm was incubated to a final concentration of $1 \mu \mathrm{M}$ in a total volume of $100 \mu \mathrm{L}$ of $50 \mathrm{mM}$ Hepes, pH7.5, $5 \mathrm{mM} \mathrm{CaCl}_{2}$, and $10 \mu \mathrm{M} \mathrm{ZnCl}$. Synthetic quenched fluorescent peptide substrates were added from a 100x stock in DMSO to a final concentration of $1 \mu \mathrm{M}$. The excitation and emission wavelengths were set at 320 and $405 \mathrm{~nm}$, respectively and the fluorescence was measured at one minute intervals for $90 \mathrm{~min}$.

\section{Subcellular localization}

The coding sequence of Sl3-MMP was amplified from pMD19-Sl3-MMP using a pair of primers Sl3-MMPGFP-F and Sl3-MMP-GFP-R (Additional file 3) and inserted into pFGC-Egfp at BamHI/XbaI sites. The recombinant plasmid pFGC-Sl3-MMP and the empty vector pFGC-Egfp were introduced into onion epidermal cells by particle bombardment method. Particle bombardment was performed with a PDS-1000 (Bio-Rad, Hercules, CA, USA) according to the manufacturer's instructions. GFP was detected $24 \mathrm{~h}$ after bombardment. Plasmolysis was achieved by treating the bombarded onion epidermal cells with $0.8 \mathrm{M}$ mannitol for $10 \mathrm{~min}$.
Microscopic observation was performed using a Zeiss LSM 780 confocal laser scanning microscope (Carl Zeiss, Germany) and representative photographs were taken.

\section{VIGS in tomato and transient expression in $\mathbf{N}$. benthamiana}

For VIGS constructs, fragments of 300-400 bp (Additional file 2) for Sl-MMPs were amplified from tomato cDNAs using gene-specific primers (Additional file 3) and cloned into pTRV2 vector [26], yielding pTRV2Sl1-MMP-pTRV2-S15-MMP. The recombinant plasmids with pTRV2-GUS (as control) were then introduced into Agrobacterium tumefaciens strain GV3101 by electroporation using GENE PULSER II Electroporation System (Bio-Rad Laboratories, Hercules, CA, USA). Agrobacteria carrying pTRV2-GUS (control) or pTRV2-Sl-MMP plasmids were grown in YEP medium $(50 \mu \mathrm{g} / \mathrm{ml}$ rifampicin, $50 \mu \mathrm{g} / \mathrm{ml}$ kanamycin and $25 \mu \mathrm{g} / \mathrm{ml}$ gentamicin) for $24 \mathrm{~h}$ with continuous shaking at $28^{\circ} \mathrm{C}$. Cells were centrifuged and resuspended in infiltration buffer $(10 \mathrm{mM}$ $\mathrm{MgCl}_{2}, 10 \mathrm{mM}$ MES, $200 \mu \mathrm{M}$ acetosyringone, pH5.7). Agrobacteria carrying pTRV2-GUS or pTRV2-Sl-MMP were mixed with agrobacteria carrying pTRV1 in a ratio of $1: 1$ and adjusted to $\mathrm{OD}_{600}=1.5$. The mixed agrobacteria suspension was infiltrated into the abaxial surface of 2-week-old seedlings using a $1 \mathrm{ml}$ needleless syringe. Efficiency of the silencing protocol was examined using phytoene desaturase (PDS) gene as a marker of silencing in tomato plants according to the protocol described previously [26]. The VIGS-infiltrated plants were allowed to grow for three weeks under same condition as mentioned above and then used for all experiments.

For transient expression in $N$. benthamiana, agrobacteria carrying pFGC-SI3-MMP or pFGC-eGFP empty vector were infiltrated into leaves of 4-week-old plants using $1 \mathrm{ml}$ needleless syringes. Leaf samples were collected 2 days after agroinfiltration for analyzing the expression level of Sl3-MMP and were used for disease assays and physiological, biochemical and molecular analyses.

\section{qRT-PCR analysis of gene expression}

Total RNA was extracted by Trizol regent (TaKaRa, Dalian, China) according to the manufacturer's instructions. RNA was treated with RNase-free DNase and then reverse-transcribed into cDNA using the PrimeScript RT regent kit (TaKaRa, Dalian, China). The obtained cDNAs were used for gene expression analysis with real time quantitative PCR. Each qPCR reaction contained $12.5 \mu \mathrm{L}$ SYBR Premix Ex Taq (TaKaRa, Dalian, China), $0.1 \mu \mathrm{g}$ cDNA and 7.5 pmol of each gene-specific primer (Additional file 3) in a final volume of $25 \mu \mathrm{L}$, and had three independent biological replicates. The qPCR was performed in a CFX96 real-time PCR detection system 
(BioRad, Hercules, CA, USA). Relative gene expression level was calculated using $2^{-\triangle \Delta C T}$ method as described [52].

\section{Western blot analysis}

Leaf discs were ground into $200 \mu \mathrm{l}$ lysis buffer $(50 \mathrm{mM}$ Tris-HCl, pH7.4, $150 \mathrm{mM} \mathrm{NaCl}, 1 \mathrm{mM}$ EDTA, $1 \mathrm{mM}$ DDT, $0.1 \%(v / v)$ Triton X-100, and $1 \times$ protease inhibitor cocktail from Sigma plus $1 \mathrm{mM}$ PMSF), followed by addition of $100 \mu \mathrm{l}$ loading buffer. The samples were boiled for $5 \mathrm{~min}$ and subsequently centrifuged at $10,000 \times g$ for $10 \mathrm{~min}$ at $4{ }^{\circ} \mathrm{C}$. Proteins in $20 \mu \mathrm{L}$ of the supernatant were separated on a $12 \%$ SDS-PAGE gel and transferred onto PVDF membrane by semi-dry transfer. Detection of GFP was performed using a polyclonal rabbit anti-GFP antibody (1:5000 dilution; GenScript, Nanjing, China) and a Horseradish peroxidase-conjugated antirabbit antibody (1:10,000 dilution; GenScript, Nanjing, China) according to the manufacturer's instructions. Proteins on PVDF membranes were detected by SuperSignal West Pico Chemiluminescent Substrate (Thermo Scientific, Rockford, IL, USA).

\section{Histochemical assays and measurement of electrolyte leakage}

Detection of $\mathrm{H}_{2} \mathrm{O}_{2}$ was performed by DAB staining [53]. Leaf samples were collected from inoculated tomato plants at $24 \mathrm{~h}$ after inoculation or $N$. benthamiana plants at $48 \mathrm{~h}$ after infiltration for transient expression. Leaves were dipped into DAB solution $(1 \mathrm{mg} / \mathrm{ml}, \mathrm{pH} 3.8)$ and incubated for $8 \mathrm{~h}$ in dark at room temperature. The DAB-treated leaves were removed, placed into acetic acid/glycerol/ethanol (1:1:1, vol/vol/vol), and boiled for $5 \mathrm{~min}$ in a water bath, followed by several changes of the solution. Subsequently, the leaves were maintained in $60 \%$ glycerol. Accumulation of $\mathrm{H}_{2} \mathrm{O}_{2}$ was visualized using a digital camera and quantified using ImageJ software (National Institutes of Health) from DAB image. Trypan blue staining to visualize cell death and $B$. cinerea hypha were performed as previously described [44]. Fresh tissue was harvested, stained, and boiled for $30 \mathrm{~s}$ in lactophenol $(10 \mathrm{~mL}$ of lactic acid, $10 \mathrm{~mL}$ of glycerol, $10 \mathrm{~mL}$ of liquid phenol, and $10 \mathrm{~mL}$ of distilled water) containing $10 \mathrm{mg}$ of trypan blue. Tissue was rapidly transferred and boiled in alcoholic lactophenol (2:1 $95 \%$ ethanol:lactophenol) for $1 \mathrm{~min}$, washed in $50 \%$ ethanol at room temperature for $2 \mathrm{~min}$, and stored in water. The stained leaves were examined using a digital camera.

Electrolyte leakage was measured as previously described [54]. Leaf discs ( $0.5 \mathrm{~cm}$ diameter) were washed in sterile double-distilled water for $30 \mathrm{~min}$, followed by incubation for $2 \mathrm{~h}$ at room temperature with gentle agitation. Electrolyte leakage from the leaf samples was evaluated by measuring ion conductivity using a conductivity meter (FE30, Mettler-Toledo Group, Switzerland).

\section{Statistical analysis}

All experiments were repeated independently three times. Data obtained from three independent experiments were subjected to statistical analysis according to the Student's $t$-test and the probability values of $p<0.05$ were considered as significant difference.

\section{Accession numbers for SI-MMPs and the defense-related genes}

The $S l-M M P$ sequences were deposited in GenBank database under the following accession numbers: S11MMP, KR081423; Sl2-MMP, KR081424; Sl3-MMP, KR081425; Sl4-MMP, KR081426; Sl5-MMP, KR081427. The defense-related genes used in this study and their GenBank accession numbers are as follows: SlRboh1, respiratory burst oxidase homolog 1 (NM_001247197); SlWfi1, whitefly-induced 1 (NM_001247342); SlAPX, ascorbate peroxidase (XM_006366063); SlGR, glutathione reductase (XM_010328522); SlCAT, catalase (XM_0 04238382); SISOD, superoxide dismutase (AF527880); PR1a, pathogenesis-related 1a (NM_001247869); PR1b, pathogenesis-related 1b (NM_001247385); SlLapA, leucine aminopeptidase (AK319505); SlPI-I, proteinase inhibitors I (M13938); SlPI-II, proteinase inhibitors II (JN091682).

\section{Availability of supporting data}

The cDNA and protein sequences of Sl-MMPs and the VIGS fragments for Sl-MMP genes used in this study are included in Additional files 1 and 2.

Phylogenetic datasets are available for download at Dryad (http://datadryad.org/; doi: 10.5061/dryad.7qh1r).

\section{Additional files}

Additional file 1: Sequences of CDNAs and proteins of SI-MMPs.

Additional file 2: Sequences of the VIGS fragments for SI-MMPs.

Additional file 3: Table S1. Primers used in this study for different purposes.

\footnotetext{
Abbreviations

ACC: 1-amino cyclopropane-1-carboxylic acid; APX: Ascorbate peroxidases; $B$. cinerea: Botrytis cinerea; CAT: Catalases; DAB: 3, 3-diaminobenzidine; dpi: Days post-inoculation; ECM: Extracellular matrix; EDTA: Ethylenediaminetetraacetic acid; GR: Glutathione reductase; MeJA: Methyl jasmonate; MMP: Matrix metalloproteinase; PDS: Phytoene desaturase; Pst: Pseudomonas syringae pv. tomato; QF: Quenched fluorescent; ROS: Reactive oxygen species; qRT-PCR: Quantitative reverse transcription PCR; SA: Salicylic acid; SOD: Superoxide dismutase; VIGS: Virus-induced gene silencing.
}

Competing interests

The authors declare that they have no competing interests. 


\section{Author's contributions}

$\mathrm{DL}$ and FS designed the experiments. DL, HZ, QS, LW, SL, YH and LH carried out most of the experiments. DL performed bioinformatics analysis. FS and $\mathrm{DL}$ wrote the paper. All authors read and approved the final manuscript.

\section{Acknowledgements}

This work was supported by the National High-Tech R \& D Program (No. 2012AA101504), the National Basic Research Program of China (2009CB119005), the National Key Technology R \& D Program of China (2011BAD12B04) and the Research Fund for the Doctoral Program of Higher Education of China (20120101110070).

Received: 7 April 2015 Accepted: 29 May 2015

Published online: 14 June 2015

\section{References}

1. Van Der Hoorn RAL. Plant proteases: From phenotypes to molecular mechanisms. Annu Rev Plant Biol. 2008;59:191-223.

2. Marino G, Funk C. Matrix metalloproteinases in plants: a brief overview. Physiol Plant. 2012;145(1):196-202.

3. Rawlings ND, Barrett AJ, Bateman A. MEROPS: The peptidase database. Nucleic Acids Res. 2010;38:D227-33

4. Rawlings ND, Barrett AJ. Introduction: Metallopeptidases and their clans. Handbook of Proteolytic Enzymes. 2004;1:231-68.

5. Massova I, Kotra LP, Fridman R, Mobashery S. Matrix metalloproteinases: Structures, evolution, and diversification. FASEB J. 1998;12(12):1075-95.

6. Gomis-Rüth FX. Structural aspects of the metzincin clan of metalloendopeptidases. Appl Biochem Biotechnol-Part B Mol Biotechnol. 2003;24(2):157-202.

7. Cauwe B, Opdenakker G. Intracellular substrate cleavage: A novel dimension in the biochemistry, biology and pathology of matrix metalloproteinases. Crit Rev Biochem Mol Biol. 2010:45(5):351-423.

8. Piccard H, Van Den Steen PE, Opdenakker G. Hemopexin domains as multifunctional liganding modules in matrix metalloproteinases and other proteins. J Leukoc Biol. 2007:81(4):870-92.

9. Page-McCaw A, Ewald AJ, Werb Z. Matrix metalloproteinases and the regulation of tissue remodelling. Nat Rev Mol Cell Biol. 2007:8(3):221-33.

10. Murphy $\mathrm{G}$, Nagase H. Progress in matrix metalloproteinase research. Mol Aspects Med. 2008;29(5):290-308.

11. Schiermeyer A, Hartenstein H, Mandal MK, Otte B, Wahner V, Schillberg S. A membrane-bound matrix-metalloproteinase from Nicotiana tabacum cv. BY-2 is induced by bacterial pathogens. BMC Plant Biol. 2009;9:83

12. Ratnaparkhe SM, Egertsdotter EMU, Flinn BS. Identification and characterization of a matrix metalloproteinase (Pta1-MMP) expressed during Loblolly pine (Pinus taeda) seed development, germination completion, and early seedling establishment. Planta. 2009;230(2):339-54.

13. Combier JP, Vernié $T$, De Billy $F$, El Yahyaoui $F$, Mathis $R$, Gamas $P$. The MtMMPL1 early nodulin is a novel member of the matrix metalloendoproteinase family with a role in Medicago truncatula infection by Sinorhizobium meliloti. Plant Physiol. 2007;144(2):703-16.

14. Hadler-Olsen E, Fadnes B, Sylte I, Uhlin-Hansen L, Winberg JO. Regulation of matrix metalloproteinase activity in health and disease. FEBS $J$. 2011;278(1):28-45

15. Maidment JM, Moore D, Murphy GP, Murphy G, Clark IM. Matrix metalloproteinase homologues from Arabidopsis thaliana. Expression and activity. J Biol Chem. 1999;274(49):34706-10.

16. Ragster L, Chrispeels MJ. Azocoll-digesting proteinases in soybean leaves: Characteristics and changes during leaf maturation. Plant Physiol. 1979;64:857-62.

17. Delorme VGR, McCabe PF, Kim DJ, Leaver CJ. A matrix metalloproteinase gene is expressed at the boundary of senescence and programmed cell death in cucumber. Plant Physiol. 2000;123(3):917-27.

18. Golldack D, Popova OV, Dietz KJ. Mutation of the matrix metalloproteinase At2-MMP inhibits growth and causes late flowering and early senescence in Arabidopsis. J Biol Chem. 2002;277(7):5541-7.

19. Liu Y, Dammann C, Bhattacharyya MK. The matrix metalloproteinase gene GmMMP2 is activated in response to pathogenic infections in soybean. Plant Physiol. 2001;127(4):1788-97.

20. Flinn BS. Plant extracellular matrix metalloproteinases. Funct Plant Biol. 2008;35(12):1183-93.
21. Pak JH, Liu CY, Huangpu J, Graham JS. Construction and characterization of the soybean leaf metalloproteinase cDNA. FEBS Lett. 1997;404(2-3):283-8.

22. Van Wart HE, Birkedal-Hansen $\mathrm{H}$. The cysteine switch: a principle of regulation of metalloproteinase activity with potential applicability to the entire matrix metalloproteinase gene family. Proc Natl Acad Sci U S A. 1990;87(14):5578-82.

23. Sekhon BS. Matrix metalloproteinases-an overview. Res Rep Biol. 2010;1:1-20.

24. Kubo T, Saito T, Fukuzawa H, Matsuda Y. Two tandemly-located matrix metalloprotease genes with different expression patterns in the Chlamydomonas sexual cell cycle. Curr Genet. 2001;40(2):136-43.

25. Kumagai MH, Donson J, Della-Cioppa G, Harvey D, Hanley K, Grill LK. Cytoplasmic inhibition of carotenoid biosynthesis with virus-derived RNA. Proc Natl Acad Sci USA. 1995;92(5):1679-83.

26. Liu Y, Schiff M, Dinesh-Kumar SP. Virus-induced gene silencing in tomato. Plant J. 2002;31(6):777-86.

27. Kawazu K, Mochizuki A, Sato Y, Sugeno W, Murata M, Seo S, et al. Different expression profiles of jasmonic acid and salicylic acid inducible genes in the tomato plant against herbivores with various feeding modes. Arthropod-Plant Interactions. 2012;6(2):221-30.

28. Mandal MK, Fischer R, Schillberg S, Schiermeyer A. Biochemical properties of the matrix metalloproteinase NtMMP1 from Nicotiana tabacum CV. BY-2 suspension cells. Planta. 2010:232(4):899-910.

29. McGeehan G, Burkhart W, Anderegg R, Becherer JD, Gillikin JW, Graham JS. Sequencing and characterization of the soybean leaf metalloproteinase: Structural and functional similarity to the matrix metalloproteinase family. Plant Physiol. 1992;99(3):1179-83.

30. Marino G, Huesgen PF, Eckhard U, Overall CM, Schroder WP, Funk C. Familywide characterization of matrix metalloproteinases from Arabidopsis thaliana reveals their distinct proteolytic activity and cleavage site specificity. Biochem J. 2014:457(2):335-46.

31. Knight CG, Willenbrock F, Murphy G. A novel coumarin-labelled peptide for sensitive continuous assays of the matrix metalloproteinases. FEBS Lett. 1992;296(3):263-6.

32. Murphy G, Nguyen Q, Cockett MI, Atkinson SJ, Allan JA, Knight CG, et al. Assessment of the role of the fibronectin-like domain of gelatinase A by analysis of a deletion mutant. J Biol Chem. 1994:269(9):6632-6.

33. Nagase $H$, Visse R, Murphy G. Structure and function of matrix metalloproteinases and TIMPs. Cardiovasc Res. 2006;69(3):562-73.

34. Cho CW, Chung E, Kim K, Soh HA, Jeong YK, Lee SW, et al. Plasma membrane localization of soybean matrix metalloproteinase differentially induced by senescence and abiotic stress. Biol Plant. 2009;53(3):461-7.

35. Nagase H. Substrate Specificity of MMPs. In: Clendeninn N, Appelt K, editors. Matrix Metalloproteinase Inhibitors in Cancer Therapy. Cancer Drug Discovery and Development: Humana Press; 2001. p. 39-66.

36. Temme N, Tudzynski P. Does Botrytis cinerea ignore $\mathrm{H}_{2} \mathrm{O}_{2}$-induced oxidative stress during infection? Characterization of Botrytis activator protein 1. Mol Plant Microbe Interact. 2009;22(8):987-98.

37. Govrin EM, Rachmilevitch S, Tiwari BS, Solomon M, Levine A. An elicitor from Botrytis cinerea induces the hypersensitive response in Arabidopsis thaliana and other plants and promotes the gray mold disease. Phytopathology. 2006;96(3):299-307.

38. Govrin EM, Levine A. The hypersensitive response facilitates plant infection by the necrotrophic pathogen Botrytis cinerea. Curr Biol. 2000;10(13):751-7.

39. Mengiste T. Plant immunity to necrotrophs. Annu Rev Phytopathol. 2012;50:267-94

40. Asselbergh B, Curvers K, Franca SC, Audenaert K, Vuylsteke M, Van Breusegem F, et al. Resistance to Botrytis cinerea in sitiens, an abscisic aciddeficient tomato mutant, involves timely production of hydrogen peroxide and cell wall modifications in the epidermis. Plant Physiol. 2007;144(4):1863-77.

41. Marino D, Dunand C, Puppo A, Pauly N. A burst of plant NADPH oxidases. Trends Plant Sci. 2012;17(1):9-15.

42. Kużniak E, Skłodowska M. Fungal pathogen-induced changes in the antioxidant systems of leaf peroxisomes from infected tomato plants. Planta. 2005;222(1):192-200.

43. Abuqamar S, Chai MF, Luo H, Song F, Mengiste T. Tomato protein kinase $1 \mathrm{~b}$ mediates signaling of plant responses to necrotrophic fungi and insect herbivory. Plant Cell. 2008;20(7):1964-83.

44. Li X, Zhang Y, Huang L, Ouyang Z, Hong Y, Zhang H, et al. Tomato SIMKK2 and SIMKK4 contribute to disease resistance against Botrytis cinerea. BMC Plant Biol. 2014;14:166. 
45. Fernandez-Pozo N, Menda N, Edwards JD, Saha S, Tecle IY, Strickler SR, et al. The Sol Genomics Network (SGN)-from genotype to phenotype to breeding. Nucleic Acids Res. 2015;43:D1036-41.

46. Altschul SF, Gish W, Miller W, Myers EW, Lipman DJ. Basic local alignment search tool. J Mol Biol. 1990;215(3):403-10.

47. Letunic I, Doerks T, Bork P. SMART 7: recent updates to the protein domain annotation resource. Nucleic Acids Res. 2012;40:D302-5.

48. Finn RD, Bateman A, Clements J, Coggill P, Eberhardt RY, Eddy SR, et al. Pfam: the protein families database. Nucleic Acids Res. 2013;42:D222-30.

49. Thompson JD, Gibson TJ, Plewniak F, Jeanmougin F, Higgins DG. The CLUSTAL_X windows interface: flexible strategies for multiple sequence alignment aided by quality analysis tools. Nucleic Acids Res. 1997;25(24):4876-82.

50. Tamura K, Stecher G, Peterson D, Filipski A, Kumar S. MEGA6: Molecular evolutionary genetics analysis version 6.0. Mol Biol Evol. 2013;30(12):2725-9.

51. Candiano G, Bruschi M, Musante L, Santucci L, Ghiggeri GM, Carnemolla B, et al. Blue silver: A very sensitive colloidal Coomassie G-250 staining for proteome analysis. Electrophoresis. 2004;25(9):1327-33.

52. Livak KJ, Schmittgen TD. Analysis of relative gene expression data using real-time quantitative PCR and the 2(-Delta Delta C(T)) Method. Methods. 2001;25(4):402-8

53. ThordalChristensen $\mathrm{H}$, Zhang ZG, Wei YD, Collinge DB. Subcellular localization of $\mathrm{H}_{2} \mathrm{O}_{2}$ in plants. $\mathrm{H}_{2} \mathrm{O}_{2}$ accumulation in papillae and hypersensitive response during the barley-powdery mildew interaction. Plant J. 1997;11(6):1187-94.

54. Choi DS, Hwang IS, Hwang BK. Requirement of the cytosolic interaction between PATHOGENESIS-RELATED PROTEIN10 and LEUCINE-RICH REPEAT PROTEIN1 for cell death and defense signaling in pepper. Plant Cell. 2012;24(4):1675-90.

\section{Submit your next manuscript to BioMed Central and take full advantage of:}

- Convenient online submission

- Thorough peer review

- No space constraints or color figure charges

- Immediate publication on acceptance

- Inclusion in PubMed, CAS, Scopus and Google Scholar

- Research which is freely available for redistribution 